\title{
MULTISHIFT VARIANTS OF THE QZ ALGORITHM WITH AGGRESSIVE EARLY DEFLATION*
}

\author{
BO KÅGSTRÖM ${ }^{\dagger}$ AND DANIEL KRESSNER ${ }^{\dagger \ddagger}$
}

\begin{abstract}
New variants of the QZ algorithm for solving the generalized eigenvalue problem are proposed. An extension of the small-bulge multishift QR algorithm is developed, which chases chains of many small bulges instead of only one bulge in each QZ iteration. This allows the effective use of level 3 BLAS operations, which in turn can provide efficient utilization of high performance computing systems with deep memory hierarchies. Moreover, an extension of the aggressive early deflation strategy is proposed, which can identify and deflate converged eigenvalues long before classic deflation strategies would. Consequently, the number of overall QZ iterations needed until convergence is considerably reduced. As a third ingredient, we reconsider the deflation of infinite eigenvalues and present a new deflation algorithm, which is particularly effective in the presence of a large number of infinite eigenvalues. Combining all these developments, our implementation significantly improves existing implementations of the QZ algorithm. This is demonstrated by numerical experiments with random matrix pairs as well as with matrix pairs arising from various applications.
\end{abstract}

Key words. generalized eigenvalue problem, generalized Schur form, QZ algorithm, multishifts, aggressive early deflation, blocked algorithms

AMS subject classifications. 65F $15,15 \mathrm{~A} 18,15 \mathrm{~A} 22,47 \mathrm{~A} 75$

DOI. $10.1137 / 05064521 \mathrm{X}$

1. Introduction. The QZ algorithm is a numerically backward stable method for computing generalized eigenvalues and deflating subspaces of small- to mediumsized regular matrix pairs $(A, B)$ with $A, B \in \mathbb{R}^{n \times n}$. It goes back to Moler and Stewart in 1973 [37] and underwent only a few modifications during the following 25 years, notably through works by Ward [47, 48], Kaufman [29], and Dackland and Kågström [12]. Nonorthogonal variants of the QZ algorithm include the LZ algorithm by Kaufman [28] and the AB algorithm for pencils by Kublanovskaya [34].

The purpose of the QZ algorithm is to compute a generalized Schur decomposition of $(A, B)$, i.e., orthogonal matrices $Q$ and $Z$ so that $S=Q^{T} A Z$ is quasi-upper triangular with $1 \times 1$ and $2 \times 2$ blocks on the diagonal, while the matrix $T=Q^{T} B Z$ is upper triangular. This decomposition provides almost everything needed to solve the generalized nonsymmetric eigenvalue problem (GNEP). Generalized eigenvalues, defined as root pairs $(\alpha, \beta)$ of the bivariate polynomial $\operatorname{det}(\beta A-\alpha B)$, can be directly computed from the diagonal blocks of $S$ and $T$, although some care must be taken to implement this computation in a safe manner; see [37, 45]. Moreover, the leading $k$ columns of the orthogonal matrices $Z$ and $Q$ span a pair of deflating subspaces [40] if the $(k+1, k)$ subdiagonal entry of the matrix $S$ vanishes. A reordering of the diagonal blocks of $S$ and $T$ can be used to compute other deflating subspaces; see [26, 25, 44].

The eigenvalues of $(A, B)$ are read off from $(S, T)$ as follows. The $2 \times 2$ diagonal blocks correspond to pairs of complex conjugate eigenvalues. The real eigenvalues

\footnotetext{
${ }^{*}$ Received by the editors November 15, 2005; accepted for publication (in revised form) by P. Benner August 21, 2006; published electronically December 21, 2006. Supported by the DFG Emmy Noether fellowship KR 2950/1-1 and by the Swedish Research Council under grant VR 621-20013284 and by the Swedish Foundation for Strategic Research under grant A3 02:128. This research was conducted using the resources of the High Performance Computing Center North (HPC2N).

http://www.siam.org/journals/simax/29-1/64521.html

${ }^{\dagger}$ Department of Computing Science and HPC2N, Umeå University, S-901 87 Umeå, Sweden (bokg@cs.umu.se, kressner@cs.umu.se).

¥Department of Mathematics, Bijenička 30, 10000 Zagreb, Croatia (kressner@math.hr).
} 
are given in pairs $\left(s_{i i}, t_{i i}\right)$ corresponding to the $1 \times 1$ diagonal blocks of $(S, T)$. The finite eigenvalues are $s_{i i} / t_{i i}$, where $t_{i i} \neq 0$. An infinite eigenvalue is represented as $\left(s_{i i}, 0\right)$ with $s_{i i} \neq 0$. If $\left(s_{i i}, t_{i i}\right) \neq(0,0)$ for all $i$, then $(A, B)$ is a regular matrix pair, or equivalently $\beta A-\alpha B$ is a regular matrix pencil. Otherwise, the matrix pair is singular and at least one $\left(s_{i i}, t_{i i}\right)$ equals $(0,0)$. These situations need extra caution, and so-called staircase-type algorithms can be used for identifying singular cases by computing a generalized upper triangular (GUPTRI) form of $(A, B)$ (e.g., see Demmel and Kågström $[13,14])$.

Three ingredients make the QZ algorithm work effectively. First, the matrix pair $(A, B)$ is reduced to Hessenberg-triangular form; i.e., orthogonal matrices $Q$ and $Z$ are computed so that $H=Q^{T} A Z$ is upper Hessenberg and $T=Q^{T} B Z$ is upper triangular. Second, a sequence of so-called implicit shifted QZ iterations is applied to $(H, T)$ in order to bring $H$ closer to (block) upper triangular form while preserving the Hessenberg-triangular form of $(H, T)$. Each of these iterations can be seen as chasing a pair of bulges from the top left to the bottom right corners along the subdiagonals of $H$ and $T$, a point of view that has been emphasized by Watkins and Elsner [49]. The third ingredient is deflation, which aims at splitting the computation of the generalized Schur form $(S, T)$ into smaller subproblems. This paper describes improvements for the latter two ingredients, QZ iterations, and deflations.

Inspired by the works of Braman, Byers, and Mathias [7] and Lang [36] for the QR algorithm, we propose multishift QZ iterations that chase a tightly coupled chain of bulge pairs instead of only one bulge pair per iteration. This allows the effective use of level 3 BLAS operations $[15,23,24]$ during the bulge chasing process, which in turn can provide efficient utilization of today's high performance computing systems with deep memory hierarchies. Tightly coupled bulge chasing has also successfully been used in the reduction of a matrix pair $\left(H_{r}, T\right)$ in block Hessenberg-triangular form, where $H_{r}$ has $r$ subdiagonals, to Hessenberg-triangular form $(H, T)$ [12].

Recently, Braman, Byers, and Mathias [6] also presented a new, advanced deflation strategy, the so-called aggressive early deflation. Combining this deflation strategy with multishift QR iterations leads to a variant of the QR algorithm, which may, for sufficiently large matrices, require less than $10 \%$ of the computing time needed by the LAPACK [2] implementation. We will show that this deflation strategy can be extended to the QZ algorithm, resulting in similar time savings.

A (nearly) singular matrix $B$ often implies that the triangular matrix $T$ of the corresponding Hessenberg-triangular form has one or more diagonal entries close to zero. Each of these diagonal entries admits the deflation of an infinite eigenvalue. Some applications, such as semidiscretized Stokes equations [42], lead to matrix pairs that have a large number of infinite eigenvalues. Consequently, a substantial amount of computational work in the QZ algorithm is spent deflating these eigenvalues. We will provide a discussion on this matter including preprocessing techniques, and we propose windowing techniques that lead to more efficient algorithms for deflating infinite eigenvalues within the QZ algorithm. This approach is conceptually close to blocked algorithms for reordering eigenvalues in standard and generalized Schur forms [32].

The rest of this paper is organized as follows. In section 2, we review and extend conventional multishift QZ iterations and provide some new insight into their numerical backward stability. Multishift variants that are based on chasing a tightly coupled chain of bulge pairs are described in section 3. In section 4, a thorough discussion on dealing with infinite eigenvalues is presented that includes preprocessing and efficient methods for deflating such eigenvalues within the QZ algorithm. Aggressive early de- 
flation for the QZ algorithm and its connection to the distance of uncontrollability for descriptor systems are studied in section 6. Computational experiments, presented in section 7, demonstrate the effectiveness of our newly developed multishift QZ algorithm with advanced deflation techniques. Finally, some concluding remarks are summarized in section 8 .

2. Conventional multishift QZ iterations. Throughout the rest of this paper we assume that the matrix pair under consideration, which will be denoted by $(H, T)$, is already in Hessenberg-triangular form. Efficient algorithms for reducing a given matrix pair to this form can be found in $[12,31]$. For the moment, we also assume that $(H, T)$ is an unreduced matrix pair; i.e., all subdiagonal entries of $H$ as well as all diagonal entries of $T$ are different from zero. The latter condition implies that only finite eigenvalues are considered.

A QZ iteration relies on a fortunate choice of $m$ shifts (or shift pairs) $\left(\mu_{1}, \nu_{1}\right)$, $\left(\mu_{2}, \nu_{2}\right), \ldots,\left(\mu_{m}, \nu_{m}\right)$ with $\mu_{i} \in \mathbb{C}$ and $\nu_{i} \in \mathbb{R}$, giving rise to the shift polynomial

$$
p\left(H T^{-1}\right)=\left(\nu_{1} H T^{-1}-\mu_{1} I_{n}\right)\left(\nu_{2} H T^{-1}-\mu_{2} I_{n}\right) \cdots\left(\nu_{m} H T^{-1}-\mu_{m} I_{n}\right) .
$$

If $x$ denotes the first column of this matrix polynomial, then the first step of an implicit shifted QZ iteration consists of choosing an orthogonal matrix $Q_{1}$ such that $Q_{1}^{T} x$ is a multiple of the first unit vector $e_{1}$. The rest of the QZ iteration consists of reducing the updated matrix pair $\left(Q_{1}^{T} H, Q_{1}^{T} T\right)$ back to Hessenberg-triangular form, without modifying the first rows of $Q_{1}^{T} H$ and $Q_{1}^{T} T$ by transformations from the left.

In the original formulation of the QZ algorithm [37], this reduction to Hessenbergtriangular form was described for $m \leq 2$, based on combinations of Givens rotations and Householder matrices. This approach has the negative side-effect that one QZ iteration with $m=2$ shifts requires more flops (floating point operations) than two QZ iterations with $m=1$ shift. Partly avoiding this increase of flops, Ward [47] proposed the so-called combination shift $Q Z$ algorithm which uses $m=1$ for real shifts and $m=2$ for complex conjugate pairs of shifts. Later on, Watkins and Elsner [49] proposed a variant solely based on Householder matrices which requires roughly $27 \%$ fewer flops than the original formulation and may employ an arbitrary number $m$ of shifts. This variant is currently implemented in the LAPACK subroutine DHGEQZ. A curiosity of this subroutine is that it still uses Ward's combination shift strategy despite the fact that two single shift QZ iterations now require roughly $9 \%$ more flops than one double shift iteration.

2.1. Householder-based variants. In the following, we describe the Householder-based variant by Watkins and Elsner in more detail. To simplify the notation, we make use of the following convention.

Definition 2.1. A Householder matrix which maps the last $n-j$ elements of a given vector $x \in \mathbb{R}^{n}$ to zero without modifying the leading $j-1$ elements is denoted by $\mathcal{H}_{j}(x)$.

Let us illustrate the first few steps of an implicit QZ iteration for $n=6, m=2$. First, a Householder matrix $\mathcal{H}_{1}(x)$ is used to map $x$, the first column of the shift polynomial defined in (2.1), to a multiple of $e_{1}$. Note that only the leading three elements of $x$ are nonzero. Hence, if $\mathcal{H}_{1}(x)$ is applied from the left to $H$ and $T$, only the first three rows (denoted by the symbols $\hat{h}$ and $\hat{t}$ below) are affected while the 
remaining rows stay unchanged (denoted by $h$ and $t$ ):

$$
(H, T) \leftarrow\left(\left[\begin{array}{llllll}
\hat{h} & \hat{h} & \hat{h} & \hat{h} & \hat{h} & \hat{h} \\
\hat{h} & \hat{h} & \hat{h} & \hat{h} & \hat{h} & \hat{h} \\
\hat{h} & \hat{h} & \hat{h} & \hat{h} & \hat{h} & \hat{h} \\
0 & 0 & h & h & h & h \\
0 & 0 & 0 & h & h & h \\
0 & 0 & 0 & 0 & h & h
\end{array}\right],\left[\begin{array}{llllll}
\hat{t} & \hat{t} & \hat{t} & \hat{t} & \hat{t} & \hat{t} \\
\hat{t} & \hat{t} & \hat{t} & \hat{t} & \hat{t} & \hat{t} \\
\hat{t} & \hat{t} & \hat{t} & \hat{t} & \hat{t} & \hat{t} \\
0 & 0 & 0 & t & t & t \\
0 & 0 & 0 & 0 & t & t \\
0 & 0 & 0 & 0 & 0 & t
\end{array}\right]\right) .
$$

Next, to avoid further fill-in in the factor $T$, the newly introduced entries $(2,1)$ and $(3,1)$ must be eliminated. Recall that we are not allowed to change the first row of $T$ by applying a transformation from the left. However, it is still possible to achieve these eliminations by applying a Householder matrix using the following simple fact.

Lemma 2.2 (see [49]). Let $T \in \mathbb{R}^{n \times n}$ be an invertible matrix. Then the first column of $T \mathcal{H}_{1}\left(T^{-1} e_{1}\right)$ is a scalar multiple of $e_{1}$.

Applying a Householder matrix from the right to eliminate several elements in one column (instead of one row) is somewhat opposite to their standard use. This motivates us to call such a matrix an opposite Householder matrix. Applying $\mathcal{H}_{1}\left(T^{-1} e_{1}\right)$ from the right yields the following diagram:

$$
(H, T) \leftarrow\left(\left[\begin{array}{cccccc}
\hat{h} & \hat{h} & \hat{h} & h & h & h \\
\hat{h}_{b} & \hat{h}_{b} & \hat{h}_{b} & h & h & h \\
\hat{h}_{b} & \hat{h}_{b} & \hat{h}_{b} & h & h & h \\
\hat{h}_{b} & \hat{h}_{b} & \hat{h}_{b} & h & h & h \\
0 & 0 & 0 & h & h & h \\
0 & 0 & 0 & 0 & h & h
\end{array}\right],\left[\begin{array}{cccccc}
\hat{t} & \hat{t} & \hat{t} & t & t & t \\
\hat{0}_{b} & \hat{t}_{b} & \hat{t}_{b} & t & t & t \\
\hat{O}_{b} & \hat{t}_{b} & \hat{t}_{b} & t & t & t \\
0_{b} & 0_{b} & 0_{b} & t & t & t \\
0 & 0 & 0 & 0 & t & t \\
0 & 0 & 0 & 0 & 0 & t
\end{array}\right]\right) .
$$

Here, we have used the subscript $b$ to designate entries that belong to the so-called bulge pair. The rest of the QZ iteration can be seen as pushing this bulge pair along the subdiagonals down to the bottom right corners until it vanishes. The next two steps consist of applying the Householder matrix $\mathcal{H}_{2}\left(\mathrm{He}_{1}\right)$ from the left and the opposite Householder matrix $\mathcal{H}_{2}\left(T^{-1} e_{2}\right)$ from the right:

$$
\begin{aligned}
& (H, T) \leftarrow\left(\left[\begin{array}{llllll}
h & h & h & h & h & h \\
\hat{h} & \hat{h} & \hat{h} & \hat{h} & \hat{h} & \hat{h} \\
\hat{0} & \hat{h} & \hat{h} & \hat{h} & \hat{h} & \hat{h} \\
\hat{0} & \hat{h} & \hat{h} & \hat{h} & \hat{h} & \hat{h} \\
0 & 0 & 0 & h & h & h \\
0 & 0 & 0 & 0 & h & h
\end{array}\right],\left[\begin{array}{llllll}
t & t & t & t & t & t \\
0 & \hat{t} & \hat{t} & \hat{t} & \hat{t} & \hat{t} \\
0 & \hat{t} & \hat{t} & \hat{t} & \hat{t} & \hat{t} \\
0 & \hat{t} & \hat{t} & \hat{t} & \hat{t} & \hat{t} \\
0 & 0 & 0 & 0 & t & t \\
0 & 0 & 0 & 0 & 0 & t
\end{array}\right]\right), \\
& (H, T) \leftarrow\left(\left[\begin{array}{llllll}
h & \hat{h} & \hat{h} & \hat{h} & h & h \\
h & \hat{h} & \hat{h} & \hat{h} & h & h \\
0 & \hat{h}_{b} & \hat{h}_{b} & \hat{h}_{b} & h & h \\
0 & \hat{h}_{b} & \hat{h}_{b} & \hat{h}_{b} & h & h \\
0 & \hat{h}_{b} & \hat{h}_{b} & \hat{h}_{b} & h & h \\
0 & 0 & 0 & 0 & h & h
\end{array}\right],\left[\begin{array}{llllll}
t & \hat{t} & \hat{t} & \hat{t} & t & t \\
0 & \hat{t} & \hat{t} & \hat{t} & t & t \\
0 & \hat{0}_{b} & \hat{t}_{b} & \hat{t}_{b} & t & t \\
0 & \hat{0}_{b} & \hat{t}_{b} & \hat{t}_{b} & t & t \\
0 & 0_{b} & 0_{b} & 0_{b} & t & t \\
0 & 0 & 0 & 0 & 0 & t
\end{array}\right]\right) .
\end{aligned}
$$

For general $m$ and $n$, the implicit shifted QZ iteration based on (opposite) Householder matrices is described in Algorithm 1. Here, the colon notation $A\left(i_{1}: i_{2}, j_{1}: j_{2}\right)$ is used to designate the submatrix of a matrix $A$ defined by rows $i_{1}$ through $i_{2}$ and columns $j_{1}$ through $j_{2}$.

Note that the shifts employed in Algorithm 1 are based on the generalized eigenvalues of the bottom right $m \times m$ submatrix pair, a choice which is sometimes called generalized Francis shifts and which ensures quadratic local convergence [49]. If $m \ll n$, a proper implementation of this algorithm requires $2(4 m+3) n^{2}+\mathcal{O}(n)$ flops for updating $H$ and $T$. In addition, $(4 m+3) n^{2}+\mathcal{O}(n)$ flops are required for updating each of the orthogonal factors $Q$ and $Z$. 


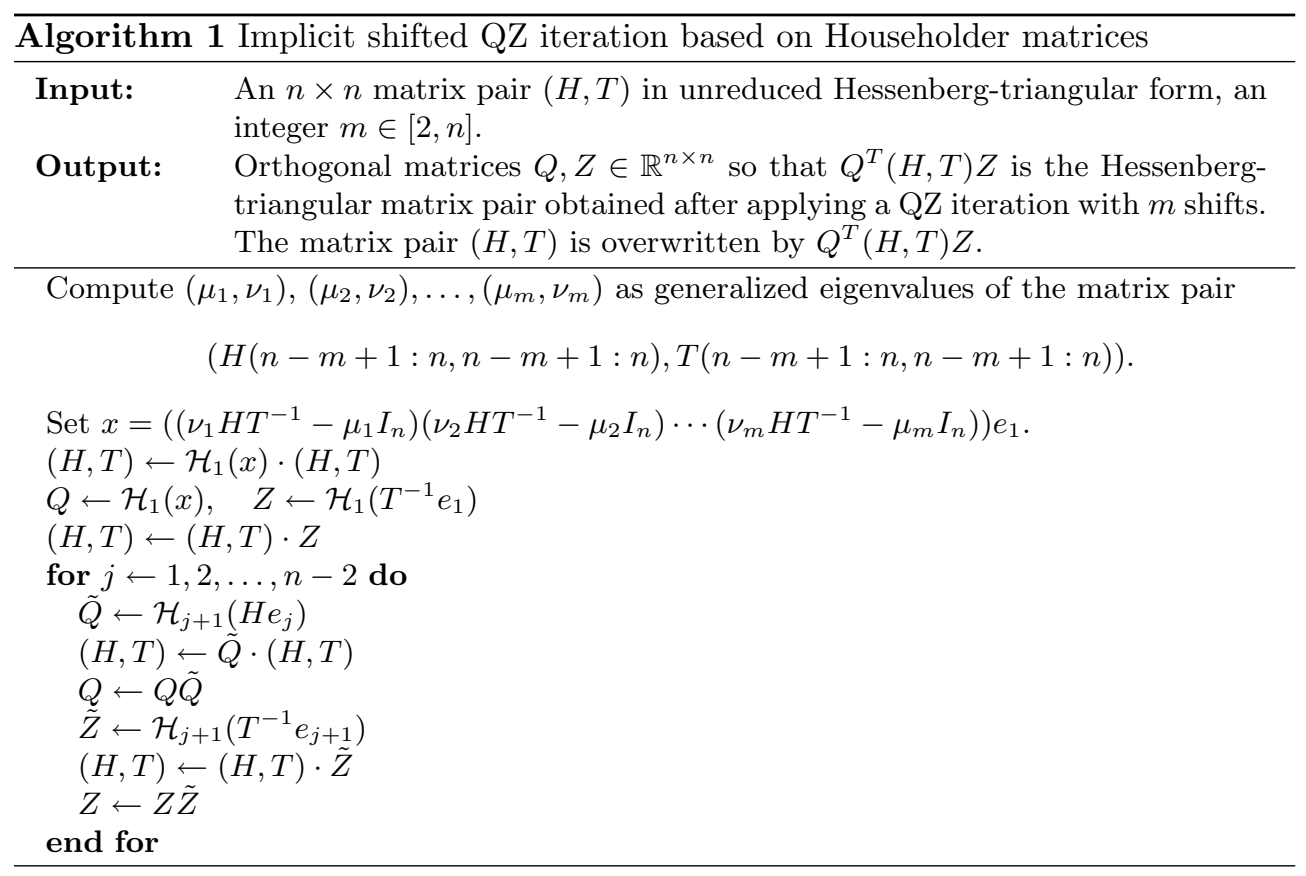

2.2. Error analysis of opposite Householder matrices. Some authors have raised concerns that the use of opposite Householder matrices could introduce numerical instabilities in the QZ algorithm; see, e.g., [12, p. 444]. Such instabilities could arise if some entries that should be zero after the application of an opposite Householder matrix are nonnegligible in finite-precision arithmetic. In the following, we provide a brief error analysis showing that such an event may not occur if some care is taken.

Without loss of generality, we can restrict the analysis to an opposite Householder matrix of the form $\mathcal{H}_{1}\left(T^{-1} e_{1}\right)$ for some nonsingular matrix $T \in \mathbb{R}^{n \times n}$. Although an ill-conditioned $T$ may severely affect the data representing $\mathcal{H}_{1}\left(T^{-1} e_{1}\right)$, it has almost no effect on the purpose of $\mathcal{H}_{1}\left(T^{-1} e_{1}\right)$, which is the introduction of zero entries. To explain this, assume that a numerically backward stable method is employed to solve the linear system $T x=e_{1}$, yielding a computed solution $\hat{x}$. This implies that $\hat{x}$ is the exact solution of a slightly perturbed system

$$
(T+F) \hat{x}=e_{1}, \quad\|F\|_{2} \leq c_{T}\|T\|_{2},
$$

where $c_{T}$ is not much larger than the unit roundoff $\mathbf{u}$ [21]. Now, consider the Householder matrix $\mathcal{H}_{1}(\hat{x})=I-\tilde{\beta} \tilde{v} \tilde{v}^{T}$, where $\tilde{\beta} \in \mathbb{R}, \tilde{v} \in \mathbb{R}^{n}$, such that $\left(I-\tilde{\beta} \tilde{v} \tilde{v}^{T}\right) \hat{x}=\tilde{\gamma} e_{1}$ for some scalar $\tilde{\gamma}$. The computation of the quantities $\tilde{\beta}, \tilde{v}$ defining $\mathcal{H}_{1}(\hat{x})$ is also subject to roundoff errors. Using standard computational methods, the computed quantities $\hat{v}, \hat{\beta}$ satisfy

$$
|\hat{\beta}-\tilde{\beta}| \leq c_{\beta}|\tilde{\beta}| \approx(4 n+8) \mathbf{u}|\tilde{\beta}|, \quad\|\hat{v}-\tilde{v}\|_{2} \leq c_{v}\|\tilde{v}\|_{2} \approx(n+2) \mathbf{u}\|\tilde{v}\|_{2} ;
$$

see $[21$, p. 365]. It follows that

$$
\begin{aligned}
\left\|T \cdot\left(I-\hat{\beta} \hat{v} \hat{v}^{T}\right) e_{1}-1 / \tilde{\gamma} \cdot e_{1}\right\|_{2} & \leq\left\|T \cdot\left(I-\tilde{\beta} \tilde{v} \tilde{v}^{T}\right) e_{1}-1 / \tilde{\gamma} \cdot e_{1}\right\|_{2} \\
& +\left(2 c_{\beta}+4 c_{v}\right)\|T\|_{2}+\mathcal{O}\left(\mathbf{u}^{2}\right) \\
& \leq\left(c_{T}+2 c_{\beta}+4 c_{v}\right)\|T\|_{2}+\mathcal{O}\left(\mathbf{u}^{2}\right) .
\end{aligned}
$$


This shows that if $\hat{x}$ is computed by a backward stable method, then the last $n-1$ elements in the first column of $T\left(I-\beta \hat{v} \hat{v}^{T}\right)$ can be set to zero without spoiling the backward stability of the QZ algorithm.

In this paper, we favor the following method for constructing opposite Householder matrices. Let $T=R Q$ be an $\mathrm{RQ}$ decomposition; i.e., the matrix $R \in \mathbb{R}^{n \times n}$ is upper triangular and $Q \in \mathbb{R}^{n \times n}$ is orthogonal. If $T$ is invertible, then $Q^{T} e_{1}$ is a scalar multiple of $T^{-1} e_{1}$ implying that $\mathcal{H}_{1}\left(Q^{T} e_{1}\right)$ is an opposite Householder matrix. Even if $T$ is singular, it can be shown that the first column of $T \cdot \mathcal{H}_{1}\left(Q^{T} e_{1}\right)$ is mapped to a multiple of $e_{1}$ :

$$
\begin{aligned}
T \cdot \mathcal{H}_{1}\left(Q^{T} e_{1}\right) & =R \cdot\left[Q \cdot \mathcal{H}_{1}\left(Q^{T} e_{1}\right)\right]=\left[\begin{array}{cc}
r_{11} & R_{12} \\
0 & R_{22}
\end{array}\right]\left[\begin{array}{cc}
\tilde{q}_{11} & 0 \\
0 & \tilde{Q}_{22}
\end{array}\right] \\
& =\left[\begin{array}{cc}
r_{11} \tilde{q}_{11} & R_{12} \tilde{Q}_{22} \\
0 & R_{22} \tilde{Q}_{22}
\end{array}\right] .
\end{aligned}
$$

RQ decompositions enjoy a favorable backward error analysis, and the constant $c_{T}$ in (2.5) can be bounded by roughly $n^{2} \mathbf{u}$; see, e.g., [21, Thm. 18.4].

2.3. Bulge pairs and shift blurring. Convergence in the implicit shifted QZ iteration typically becomes manifest in the bottom right corner of the matrix pair; often the $m$ th-last subdiagonal entry of $H$ converges to zero. As a QZ iteration can be interpreted as chasing a bulge pair from the top left corner to the bottom right corner of $(H, T)$, the question arises how the information contained in the shifts is passed during this chasing process. Watkins [53] discovered a surprisingly simple relationship; the intended shifts are the finite eigenvalues of the bulge pairs.

To explain this in more detail, suppose that the implicit shifted QZ iteration with $m$ shifts, Algorithm 1, is applied to $(H, T) \in \mathbb{R}^{n \times n} \times \mathbb{R}^{n \times n}$ with $n>m$. As before, we assume that $(H, T)$ is in unreduced Hessenberg-triangular form but we do not assume that $T$ is nonsingular; only the part used for the shift computation (the trailing $m \times m$ principal submatrix of $T$ ) and the part involved in the introduction of the bulge pair (the leading $m \times m$ principal submatrix of $T$ ) are required to be nonsingular. Let $x$ be a multiple of the first column of the shift polynomial defined in (2.1). The initial bulge pair is the matrix pair $\left(B_{0}^{(H)}, B_{0}^{(T)}\right)$, where

$$
\begin{aligned}
B_{0}^{(H)}=[x(1: m+1), H(1: m+1: 1: m)] & =\left[\begin{array}{cccc}
x_{1} & h_{11} & \cdots & h_{1 m} \\
x_{2} & h_{21} & \ddots & \vdots \\
\vdots & & \ddots & h_{m m} \\
x_{m+1} & 0 & & h_{m+1, m}
\end{array}\right], \\
B_{0}^{(T)}=[0, T(1: m+1: 1: m)] & =\left[\begin{array}{cccc}
0 & t_{11} & \cdots & t_{1 m} \\
0 & 0 & \ddots & \vdots \\
\vdots & & \ddots & t_{m m} \\
0 & 0 & \cdots & 0
\end{array}\right]
\end{aligned}
$$

THEOREM 2.3 (see [53]). If the leading $m \times m$ principal submatrix of $T$ is nonsingular, then the shifts $\left(\sigma_{1}, 1\right), \ldots,\left(\sigma_{m}, 1\right)$ are the finite eigenvalues of the initial bulge $\operatorname{pair}\left(B_{0}^{(H)}, B_{0}^{(T)}\right)$.

During the course of a QZ iteration, a bulge pair is first created at the top left corners and then chased down to the bottom right corners. Let $\left(H^{(j)}, T^{(j)}\right)$ denote 
the updated matrix pair $(H, T)$ obtained after the bulge pair has been chased $j-1$ steps, which amounts to applying $j-1$ loops of Algorithm 1. Then, the $j$ th bulge pair $\left(B_{j}^{(H)}, B_{j}^{(T)}\right)$ is given by

$$
\begin{aligned}
& B_{j}^{(H)}=H^{(j)}(j+1: j+m+1, j: j+m+1), \\
& B_{j}^{(T)}=T^{(j)}(j+1: j+m+1, j: j+m+1),
\end{aligned}
$$

which corresponds to the submatrices designated by the subscript $b$ in (2.3)-(2.4).

THEOREM 2.4 (see [53]). If the mth leading principal submatrix of $T$ is nonsingular, then the shifts $\sigma_{1}, \ldots, \sigma_{m}$ are the finite eigenvalues of the $j$ th bulge pair $\left(B_{j}^{(H)}, B_{j}^{(T)}\right)$.

Note that the definition of a bulge pair is only possible for $j \leq n-m-1$, since otherwise (2.6) refers to entries outside of $H^{(j)}$ and $T^{(j)}$. This issue can be resolved by adding virtual rows and columns to the matrix pair $\left(H^{(j)}, T^{(j)}\right)$; see [53]. Theorem 2.4 can be extended to the case $j>n-m-1$.

Early attempts to improve the performance of the QR algorithm focused on using shift polynomials of high degree [4], leading to medium-order Householder matrices during the QR iteration and enabling the efficient use of WY representations. This approach, however, has proved disappointing due to the fact that the convergence of such a large-bulge multishift QR algorithm is severely affected by roundoff errors [16]. This effect is caused by shift blurring: with increasing $m$ the eigenvalues of the bulge pairs, which should represent the shifts in exact arithmetic, often become extremely sensitive to perturbations [51, 52,33]. Already for moderate $m$, say, $m \geq 15$, the shifts may be completely contaminated by roundoff errors during the bulge chasing process. Not surprisingly, we made similar observations in numerical experiments with implicit shifted QZ iterations, which also suffer from shift blurring.

3. Multishift QZ iterations based on tightly coupled tiny bulge pairs. The trouble with shift blurring can be avoided by developing variants of the implicit shifted QZ algorithm that still rely on a large number of simultaneous shifts but chase several tiny bulge pairs instead of one large bulge pair. Such ideas have already been successfully applied to the $\mathrm{QR}$ algorithm; see, e.g., $[7,36]$ and the references therein. In this section, we describe an extension of the work by Braman, Byers, and Mathias [7] to the QZ algorithm.

For the purpose of describing this new tiny-bulge multishift QZ algorithm, let $m$ denote the number of simultaneous shifts to be used in each QZ iteration and let $n_{s}$ denote the number of shifts contained in each bulge pair. It is assumed that $m$ is an integer multiple of $n_{s}$. To avoid shift blurring phenomena we use tiny values for $n_{s}$, say, $n_{s}=2$ or $n_{s}=4$.

Our algorithm performs an implicit shifted QZ iteration with $m$ generalized Francis shifts to a Hessenberg-triangular matrix pair $(H, T)$ and consists of three stages, which are described in more detail below. First, a tightly coupled chain of $m / n_{s}$ bulge pairs is bulge-by-bulge introduced in the top left corners of $H$ and $T$. Second, the whole chain at once is chased down along the subdiagonal until the bottom bulge pair reaches the bottom right corners of $H$ and $T$. Finally, all bulge pairs are bulge-by-bulge chased off this corner.

3.1. Introducing a chain of bulge pairs. The tiny-bulge multishift QZ algorithm begins with introducing $m / n_{s}$ bulge pairs in the top left corner of the matrix pair $(H, T)$. Every bulge pair contains a set of $n_{s}$ shifts. It is assumed that the $\left(\left(m / n_{s}\right)\left(n_{s}+1\right)-1\right)$ th leading principal submatrix of $T$ is nonsingular. The first 

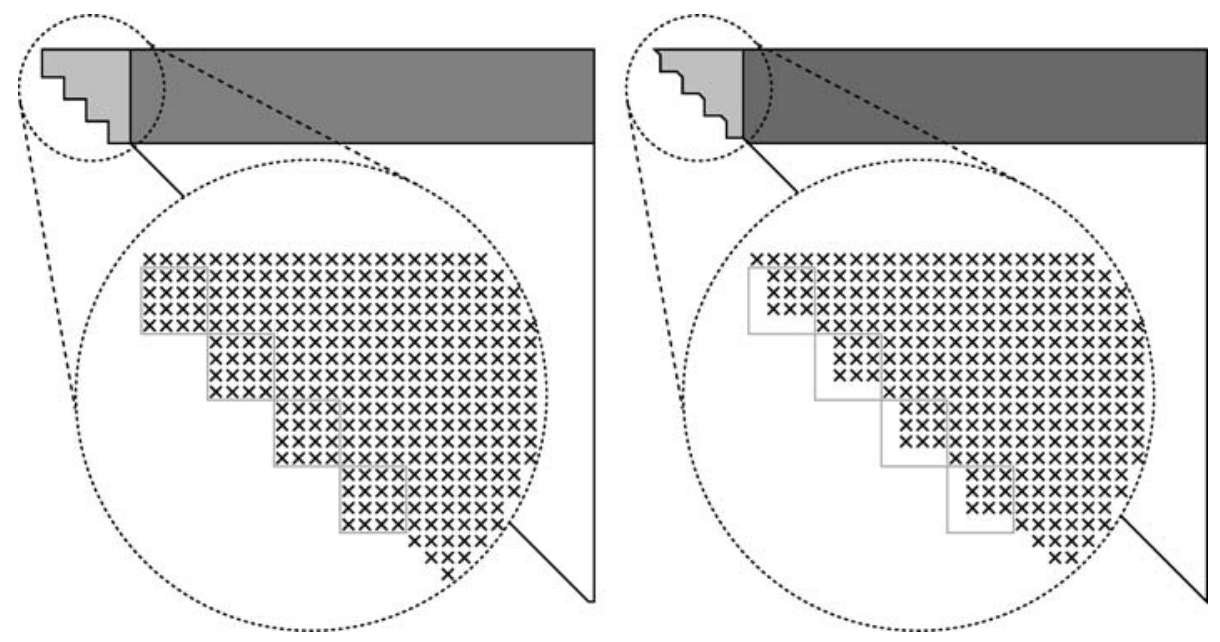

FIG. 3.1. Introducing a chain of $m / n_{s}=4$ tightly coupled bulge pairs, each of which contains $n_{s}=3$ shifts.

bulge pair is introduced by applying an implicit QZ iteration with $n_{s}$ shifts and interrupting the bulge chasing process as soon as the bottom right corner of the bulge in $H$ touches the $\left(p_{h}-1, p_{h}\right)$ subdiagonal entry of $H$, where $p_{h}=\left(m / n_{s}\right)\left(n_{s}+1\right)+1$. The next bulge pair is chased until the bottom right corner of the bulge in $H$ touches the $\left(p_{h}-n_{s}-2, p_{h}-n_{s}-1\right)$ subdiagonal entry. This process is continued until all $m / n_{s}$ bulge pairs are introduced; see Figure 3.1. Note that only the submatrices marked light gray in Figure 3.1 must be updated during the bulge chasing process. To update the remaining parts (marked dark gray), all orthogonal transformations from the left are accumulated into a $p_{h} \times p_{h}$ matrix $U$ and applied in terms of general matrix-matrix multiply (GEMM) operations:

$$
\begin{aligned}
H\left(1: p_{h},\left(p_{h}+1\right): n\right) & \leftarrow U^{T} \cdot H\left(1: p_{h},\left(p_{h}+1\right): n\right), \\
T\left(1: p_{h},\left(p_{h}+1\right): n\right) & \leftarrow U^{T} \cdot T\left(1: p_{h},\left(p_{h}+1\right): n\right) .
\end{aligned}
$$

3.2. Chasing a chain of bulge pairs. In each step of the tiny-bulge multishift QZ algorithm, the chain of bulge pairs is chased $k$ steps downward. Before the first step, this chain resides in columns/rows $p_{l}: p_{h}$ with $p_{l}=1, p_{h}=\left(m / n_{s}\right)\left(n_{s}+1\right)+1$ as above. Before the next step, we have $p_{l}=1+k, p_{h}=\left(m / n_{s}\right)\left(n_{s}+1\right)+1+k$, and so on.

The whole chain is chased in a bulge-by-bulge and bottom-to-top fashion. One such step is illustrated in Figure 3.2. Again, only the principal submatrices marked light gray in Figure 3.2 must be updated during the bulge chasing process. All transformations from the left and from the right are accumulated into orthogonal matrices $U$ and $V$, respectively. Then, GEMM operations can be used to update the rest of the matrix pair (marked dark gray in Figure 3.2):

$$
\begin{aligned}
H\left(p_{l}: p_{h}+k,\left(p_{h}+1\right): n\right) & \leftarrow U^{T} \cdot H\left(p_{l}: p_{h}+k,\left(p_{h}+1\right): n\right), \\
T\left(p_{l}: p_{h}+k,\left(p_{h}+1\right): n\right) & \leftarrow U^{T} \cdot T\left(p_{l}: p_{h}+k,\left(p_{h}+1\right): n\right), \\
H\left(1: p_{l}-1, p_{l}: p_{h}+k\right) & \leftarrow H\left(1: p_{l}-1, p_{l}: p_{h}+k\right) \cdot V, \\
T\left(1: p_{l}-1, p_{l}: p_{h}+k\right) & \leftarrow T\left(1: p_{l}-1, p_{l}: p_{h}+k\right) \cdot V .
\end{aligned}
$$



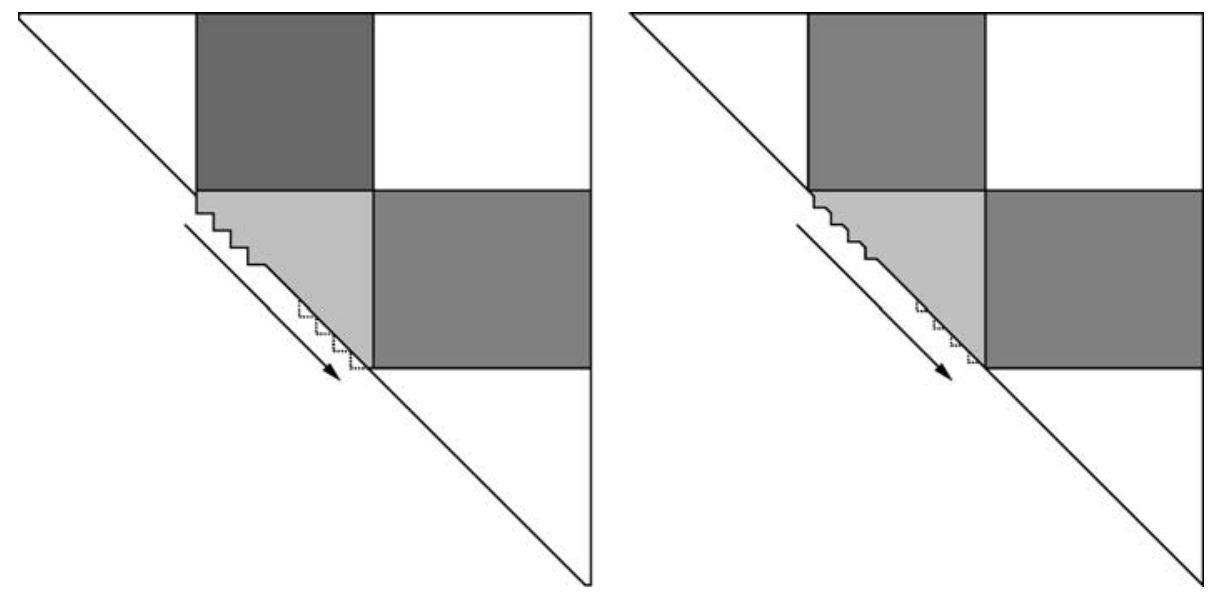

FIG. 3.2. Chasing a chain of $m / n_{s}=4$ tightly coupled bulge pairs.

Note that both matrices, $U$ and $V$, have the following block structure:

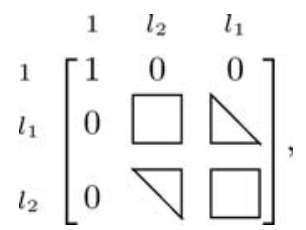

where $l_{1}=\left(m / n_{s}\right)\left(n_{s}+1\right)-n_{s}$ and $l_{2}=k+n_{s}$. If this structure is largely ignored, applying $U$ or $V$ amounts to a single GEMM with one of the factors being an $\left(l_{1}+l_{2}\right) \times\left(l_{1}+l_{2}\right)$ matrix. If, on the other hand, the triangular block structure is fully exploited, applying $U$ or $V$ amounts to two triangular matrix-matrix multiplies (TRMMs), one with an $l_{1} \times l_{1}$ factor and the other with an $l_{2} \times l_{2}$ factor, as well as two rectangular GEMMs, one with an $l_{1} \times l_{2}$ factor and the other with an $l_{2} \times l_{1}$ factor. The ratio between the flops needed by these two options is $1+\left(l_{1}^{2}+l_{2}^{2}\right) /\left(l_{1}^{2}+l_{2}^{2}+4 l_{1} l_{2}\right)$. Following the suggestion in [7], we set the number of steps the bulge chain is chased to $k=3 / 2 m$, leading to $l_{2} \approx 3 / 2 l_{1}$. In this case, exploiting the triangular block structure reduces the number of flops by $26 \%$. Whether this reduction leads to an actual saving of execution time depends on the performance of TRMM relative to GEMM, which may vary depending on BLAS implementations used for the target architecture and actual matrix sizes (e.g., see [23, 24]). A recent report [19] has identified computing environments for which TRMM performs significantly worse than GEMM, especially for the matrix dimensions arising in our application. In such a setting, it is more favorable to apply $U$ or $V$ with a single GEMM. However, many BLAS implementations, including the one proposed in [19], contain TRMM operations that perform well in comparison to GEMM. In this case, it is often possible to turn the flop reduction offered by the block triangular structure into an actual decrease of execution time.

As for the tiny-bulge multishift QR algorithm, we have to be aware of so-called vigilant deflations $[7,50]$, i.e., zero or tiny subdiagonal elements in $H$ that arise during the chasing process. In order to preserve the information contained in the bulge pairs, the chain of bulge pairs must be reintroduced in the row in which the zero appears. Fortunately, we do not have to be aware of zero or tiny subdiagonal elements in $T$, since the bulge pairs are properly passed through infinite eigenvalues; see section 4.4. 
After a certain number of steps, the bottom bulge pair of the chain reaches the bottom right corners of the matrix pair. As soon as this happens, the whole chain is bulge-by-bulge chased off this corner, similarly to the introduction of bulge pairs.

3.3. Classic deflation of finite eigenvalues. The goal of (multishift) QZ iterations is to drive the subdiagonal entries of the Hessenberg matrix in $(H, T)$ to zero while preserving the upper triangular shape of $T$. Once a subdiagonal entry $h_{k+1, k}$ is considered zero, the problem is deflated into two smaller problems:

$$
\left(\left[\begin{array}{cc}
H_{11} & H_{12} \\
0 & H_{22}
\end{array}\right],\left[\begin{array}{cc}
T_{11} & T_{12} \\
0 & T_{22}
\end{array}\right]\right) \text {. }
$$

Afterward, the (multishift) QZ iteration is applied separately to the $k \times k$ and ( $n-$ $k) \times(n-k)$ matrix pairs $\left(H_{11}, T_{11}\right)$ and $\left(H_{22}, T_{22}\right)$, respectively.

In the original formulation of the QZ algorithm [37] and the current implementation in LAPACK, a subdiagonal entry $h_{k+1, k}$ is considered zero if

$$
\left|h_{k+1, k}\right| \leq \mathbf{u}\|H\|_{F} .
$$

A more conservative criterion, in the spirit of the LAPACK implementation of the QR algorithm, is to consider $h_{k+1, k}$ zero if

$$
\left|h_{k+1, k}\right| \leq \mathbf{u}\left(\left|h_{k, k}\right|+\left|h_{k+1, k+1}\right|\right) \text {. }
$$

It is known for standard eigenvalue problems that, especially in the presence of graded matrices, the use of the criterion (3.3) gives higher accuracy in the computed eigenvalues [41]. We have observed similar accuracy improvements for the QZ algorithm when using (3.3) in favor of (3.2). We have also encountered examples where both criteria give similar accuracy but with slightly shorter execution times for (3.2) due to earlier deflations.

4. Dealing with infinite eigenvalues. If the degree $p$ of the polynomial det $(\beta A-\alpha B)$ is less than $n$ then the matrix pair $(A, B)$ is said to have $n-p$ infinite eigenvalues. The relationship between infinite eigenvalues and the QZ algorithm is subtle and calls for caution. In finite-precision arithmetic, the QZ algorithm may utterly fail to correctly identify infinite eigenvalues, especially if the index of the matrix pair, defined as the size of the largest Jordan block associated with an infinite eigenvalue [17], is larger than one [37]. In the context of differential-algebraic equations (DAEs), the index of $(A, B)$ corresponds to the index of the DAE $B \dot{x}=A x+f$. Many applications, such as multibody systems and electrical circuits, lead to DAEs with index at least two; see, e.g., [8, 39, 43].

If the matrix pair $(A, B)$ has an infinite eigenvalue then the matrix $B$ is singular. This implies that at least one of the diagonal entries in the upper triangular matrix $T$ in the Hessenberg-triangular form $(H, T)$ and in the generalized Schur form $(S, T)$ is zero, and vice versa. In finite-precision arithmetic, zero diagonal entries are spoiled by roundoff errors. While a tiny zero diagonal entry of $T$ implies that $T$ is numerically singular, the converse is generally not true. There are well-known examples of upper triangular matrices that are numerically singular but have diagonal entries that are not significantly smaller than the norm of the matrix [18, Ex. 5.5.1].

In such cases, much more reliable decisions on the nature of infinite eigenvalues can be met using algorithms that reveal Kronecker structures, such as GUPTRI [13, 14]. In some cases, infinite eigenvalues can be cheaply and reliably deflated by exploiting the structure of $A$ and $B$. 
4.1. Preprocessing deflation of infinite eigenvalues. Given a regular matrix pair $(A, B)$ with infinite eigenvalues corresponding to several Jordan blocks, the QZ algorithm will typically compute eigenvalue pairs $\left(\alpha_{i}, \beta_{i}\right)$ with $\beta_{i}$ nonzero. Moreover, otherwise well-conditioned eigenvalues can be affected by perturbations from these defective infinite eigenvalues; e.g., they may coincide or appear in clusters of eigenvalues corresponding to computed infinite as well as finite eigenvalues. In the following, we briefly describe two preprocessing techniques for handling such situations.

Exploiting staircase algorithms. Without having any knowledge of the Jordan structure of the infinite eigenvalue, in principle, the only reliable and robust way to identify all infinite eigenvalues is to apply a preprocessing step with a staircase-type algorithm.

By applying the GUPTRI algorithm $[13,14,27]$ to a regular pair $(A, B)$ with infinite eigenvalues, we get

$$
U^{T}(A, B) V=\left(\left[\begin{array}{cc}
A_{11} & A_{12} \\
0 & A_{\mathrm{inf}}
\end{array}\right],\left[\begin{array}{cc}
B_{11} & B_{12} \\
0 & B_{\mathrm{inf}}
\end{array}\right]\right)
$$

where $U$ and $V$ are orthogonal transformation matrices, $\left(A_{\text {inf }}, B_{\text {inf }}\right)$ reveals the Jordan structure of the infinite eigenvalue, and $\left(A_{11}, B_{11}\right)$ is a matrix pair with only finite eigenvalues.

Let us illustrate the GUPTRI form (4.1) with a small example. We consider a $7 \times 7$ pair $(A, B)$ with three finite eigenvalues and an infinite eigenvalue of multiplicity four corresponding to two nilpotent Jordan blocks $N_{1}$ and $N_{3}$. The infinite eigenvalue is both derogatory and defective, since it has more than one eigenvector (two Jordan blocks) but lacks a full setting of eigenvectors (four Jordan blocks). Then $\left(A_{\text {inf }}, B_{\text {inf }}\right)$ has the following schematic staircase form:

$$
\left(A_{\text {inf }}, B_{\text {inf }}\right)=\left(\left[\begin{array}{c|c|cc}
\mathbf{z} & y & x & x \\
\hline 0 & \mathbf{y} & x & x \\
\hline 0 & 0 & \mathbf{x} & \mathbf{x} \\
0 & 0 & 0 & \mathbf{x}
\end{array}\right],\left[\begin{array}{c|c|cc}
0 & \mathbf{y} & x & x \\
\hline 0 & 0 & \mathbf{x} & \mathbf{x} \\
\hline 0 & 0 & 0 & 0 \\
0 & 0 & 0 & 0
\end{array}\right]\right) .
$$

The bold numbers $\mathbf{x}, \mathbf{y}, \mathbf{z}$ in $A_{\text {inf }}$ represent diagonal blocks of full rank, and the $\mathbf{x}$ and $\mathbf{y}$ in $B_{\text {inf }}$ represent superdiagonal blocks of full row rank. Outgoing from the bottom right corner of $B_{\text {inf }}$, the sizes of the diagonal blocks (stairs) $w=(2,1,1)$ are the Weyr characteristics of the infinite eigenvalue. These indices relate to the dimensions of the nullspaces $\mathcal{N}\left(B^{j}\right)$ such that $\sum_{k=1}^{j} w_{k}=\operatorname{dim} \mathcal{N}\left(B^{j}\right)$ for $j=1,2,3$. In other words, $w_{j}$ is the number of Jordan blocks of size $\geq j$. Now, the infinite Jordan structure can be read off from $w$ giving the Segre characteristics $s=(3,1)$, where $s_{1}$ is the size of the largest Jordan block, $s_{2}$ is the size of the second largest block, and so on. Both $w$ and $s$ sum up to the algebraic multiplicity and $w_{1}$ is the geometric multiplicity of the infinite eigenvalue.

After such a preprocessing deflation of the infinite eigenvalues of $(A, B)$, we apply the QZ algorithm to the matrix pair $\left(A_{11}, B_{11}\right)$ in (4.1). For more introductory material on singular matrix pairs and the GUPTRI form see [27] and the references therein.

Exploiting knowledge of structure. In some cases, infinite eigenvalues can be reliably deflated by taking into account knowledge on the structure of the matrices $A$ and $B$. If this is feasible by orthogonal transformations, this is the recommended way 
of dealing with infinite eigenvalues, as the decision which eigenvalues are considered infinite is not affected by roundoff error. In the context of DAEs, several frameworks have been developed that can help identify and exploit such structures; see, e.g., [20, 35]. The following example is closely related to work by Stykel [42], in which $(A, B)$ arises from a semidiscretized Stokes equation.

Example 4.1. Consider $A=\left[\begin{array}{cc}K & L \\ L^{T} & 0\end{array}\right]$ and $B=\left[\begin{array}{cc}M & 0 \\ 0 & 0\end{array}\right]$, where $L$ is an $m \times(n-m)$ matrix of full column rank $(n \leq 2 m)$ and $M$ is an $m \times m$ symmetric positive definite matrix. By a QR decomposition of $L$ we may transform the matrix pair $(A, B)$ to

$$
(A, B) \leftarrow\left(\left[\begin{array}{ccc}
K_{11} & K_{12} & L_{1} \\
K_{21} & K_{22} & 0 \\
L_{1}^{T} & 0 & 0
\end{array}\right],\left[\begin{array}{ccc}
M_{11} & M_{12} & 0 \\
M_{21} & M_{22} & 0 \\
0 & 0 & 0
\end{array}\right]\right),
$$

where $L_{1}$ is an $m \times m$ invertible matrix. The submatrix $M_{22}$ is again symmetric positive definite, which in particular yields its invertibility. By a simple block permutation, $A$ and $B$ can be transformed to block upper triangular form,

$$
(A, B) \leftarrow\left(\left[\begin{array}{ccc}
L_{1} & K_{12} & K_{11} \\
0 & K_{22} & K_{21} \\
0 & 0 & L_{1}^{T}
\end{array}\right],\left[\begin{array}{ccc}
0 & M_{12} & M_{11} \\
0 & M_{22} & M_{21} \\
0 & 0 & 0
\end{array}\right]\right) .
$$

Thus, the eigenvalues of the matrix pair $\left(K_{22}, M_{22}\right)$ constitute the finite eigenvalues of $(A, B)$.

4.2. Deflation of infinite eigenvalues within the QZ algorithm. Although preprocessing is the preferable way of dealing with infinite eigenvalues, there can be good reasons to let the QZ algorithm do this job, particularly if the reliable detection of infinite eigenvalues is not a major concern. One reason is that computing a GUPTRI form is quite a costly procedure [14]. This and the following two subsections are concerned with existing and new approaches to deflate infinite eigenvalues that are signaled by tiny diagonal entries of the matrix $T$ in a Hessenberg-triangular matrix pair $(H, T)$.

For testing the smallness of a diagonal entry $t_{j j}$ we may, similar to (3.2)-(3.3), either use the norm-wise criterion

$$
\left|t_{j j}\right| \leq \mathbf{u} \cdot\|T\|_{F}
$$

as implemented in the LAPACK routine DHGEQZ, or the neighbor-wise criterion $\left|t_{j j}\right| \leq$ $\mathbf{u} \cdot\left(\left|t_{j-1, j}\right|+\left|t_{j, j+1}\right|\right)$. The latter criterion might help avoid artificial infinite eigenvalues caused by a poor scaling of the matrix pair. Let us briefly sketch the procedure developed by Moler and Stewart [37] for deflating an infinite eigenvalue after $t_{j j}$ has been set to zero, for the case $n=5$ and $j=3$ :

$$
(H, T)=\left(\left[\begin{array}{lllll}
h & h & h & h & h \\
h & h & h & h & h \\
0 & h & h & h & h \\
0 & 0 & h & h & h \\
0 & 0 & 0 & h & h
\end{array}\right],\left[\begin{array}{lllll}
t & t & t & t & t \\
0 & t & t & t & t \\
0 & 0 & 0 & t & t \\
0 & 0 & 0 & t & t \\
0 & 0 & 0 & 0 & t
\end{array}\right]\right) .
$$

First, a Givens rotation is applied to columns 2 and 3 to annihilate $t_{22}$, followed by a Givens rotation acting on rows 3 and 4 to annihilate the newly introduced nonzero entry $h_{42}$ :

$$
(H, T) \leftarrow\left(\left[\begin{array}{ccccc}
h & \hat{h} & \hat{h} & h & h \\
h & \hat{h} & \hat{h} & h & h \\
0 & \hat{h} & \hat{h} & \hat{h} & \hat{h} \\
0 & \hat{0} & \hat{h} & \hat{h} & \hat{h} \\
0 & 0 & 0 & h & h
\end{array}\right],\left[\begin{array}{ccccc}
t & \hat{t} & \hat{t} & t & t \\
0 & \hat{0} & \hat{t} & t & t \\
0 & 0 & 0 & \hat{t} & \hat{t} \\
0 & 0 & 0 & \hat{t} & \hat{t} \\
0 & 0 & 0 & 0 & t
\end{array}\right]\right) .
$$


In a similar manner, the two zero diagonal entries in $T$ are pushed one step upward:

$$
(H, T) \leftarrow\left(\left[\begin{array}{ccccc}
\hat{h} & \hat{h} & h & h & h \\
\hat{h} & \hat{h} & \hat{h} & \hat{h} & \hat{h} \\
\hat{0} & \hat{h} & \hat{h} & \hat{h} & \hat{h} \\
0 & 0 & h & h & h \\
0 & 0 & 0 & h & h
\end{array}\right],\left[\begin{array}{lllll}
\hat{0} & \hat{t} & t & t & t \\
0 & 0 & \hat{t} & \hat{t} & \hat{t} \\
0 & 0 & \hat{t} & \hat{t} & \hat{t} \\
0 & 0 & 0 & t & t \\
0 & 0 & 0 & 0 & t
\end{array}\right]\right)
$$

Finally, a Givens rotation acting on rows 1 and 2 is used to deflate the infinite eigenvalue at the top left corner:

$$
(H, T) \leftarrow\left(\left[\begin{array}{c|cccc}
\hat{h} & \hat{h} & \hat{h} & \hat{h} & \hat{h} \\
\hline \hat{0} & \hat{h} & \hat{h} & \hat{h} & \hat{h} \\
0 & h & h & h & h \\
0 & 0 & h & h & h \\
0 & 0 & 0 & h & h
\end{array}\right],\left[\begin{array}{l|llll}
0 & \hat{t} & \hat{t} & \hat{t} & \hat{t} \\
\hline 0 & \hat{t} & \hat{t} & \hat{t} & \hat{t} \\
0 & 0 & t & t & t \\
0 & 0 & 0 & t & t \\
0 & 0 & 0 & 0 & t
\end{array}\right]\right)
$$

The outlined procedure requires roughly $6 j n$ flops for updating each of the factors $H, T, Q$, and $Z$. If $j>n / 2$, it is cheaper to push the infinite eigenvalue to the bottom right corner.

4.3. Windowing techniques for deflating infinite eigenvalues. The algorithm described in the previous subsection performs $\mathcal{O}(j n)$ flops while accessing $\mathcal{O}(j n)$ memory, making it costly in terms of execution time on computing systems with deep memory hierarchies. If the dimension of the matrix pair is large and many infinite eigenvalues are to be deflated, this degrades the overall performance of the multishift QZ algorithm. A higher computation/communication ratio can be attained by using windowing techniques similar to those proposed in $[5,12,32]$. In the following, we illustrate such an algorithm, conceptually close to a recently presented block algorithm for reordering standard and generalized Schur forms [32].

Consider a matrix pair $(H, T)$ in Hessenberg-triangular form, where the 9th and the 16th diagonal entries of $T$ are zero; see Figure 4.1(a). Both zero entries will be pushed simultaneously in a window-by-window fashion to the top left corner. The first step consists of pushing the lower zero diagonal entry to the top left corner of the 8-by- 8 window marked by the light gray area in Figure 4.1(b). This creates zero diagonal entries at positions 11 and 12 . Note that it makes little sense to push one step further; the leading zero at position 10 would be destroyed when pushing the zero diagonal entry at position 9. During this procedure, only the entries of $H$ and $T$ that reside within the window are updated and the data representing the performed Givens rotations is pipelined; see [32] for more details. Afterward, the pipelined transformations are applied to the parts outside the window marked by dark gray areas in Figure 4.1(b) as well as to the corresponding parts of the transformation matrices $Q$ and $Z$. To maintain locality of the memory reference pattern, rows are updated in stripes of $n_{b}$ columns (in the computational environments we considered, choosing $n_{b}=32$ was nearly optimal). The next window contains the diagonal positions $5, \ldots, 12$; see Figure 4.1(c). The zeros at positions 9 and 11 are subsequently pushed to positions 5 and 7 , respectively. Again, the update of parts outside the window as well as the update of the transformation matrices are delayed as described above. The last 8-by- 8 window resides in the top left corner and yields the deflation of two infinite eigenvalues; see Figure 4.1(d).

Note that we have only provided the generic picture; pushing a zero diagonal entry in $T$ may leave "traces" in the form of additional zero diagonal entries. A proper implementation of the windowing algorithm has to take care of such events. 
(a)

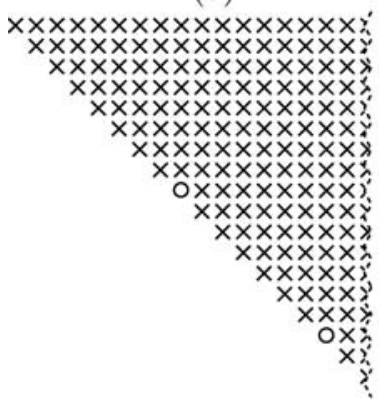

(c)

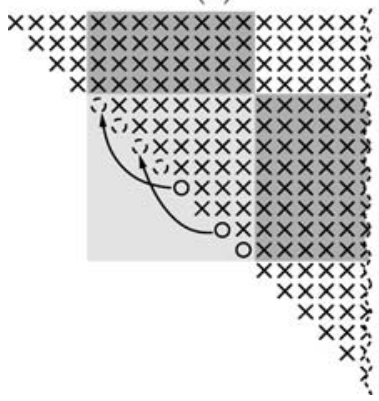

(b)

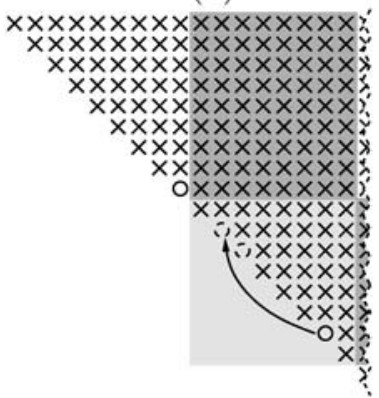

(d)

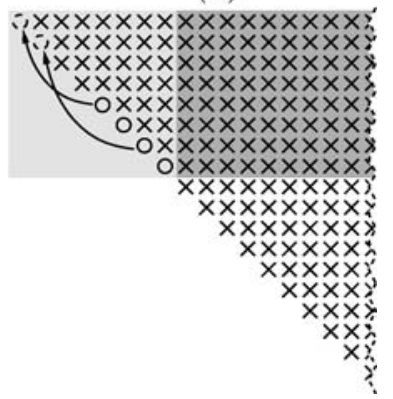

FIG. 4.1. Illustration of windowing algorithm for deflating two infinite eigenvalues. Only the T-matrix of $(H, T)$ is shown.

Moreover, to achieve optimal performance, the number of zero diagonal entries to be simultaneously pushed and the window size should be significantly larger than those chosen in the descriptive example; see section 7.2.

4.4. Infinite eigenvalues and multishift $\mathrm{QZ}$ iterations. An important observation made in [53] is that the shift transmission mechanism works even if $T$ is singular, provided that this singularity does not affect the generalized Francis shifts or the definition of the first column of the shift polynomial. In fact, Theorem 2.4 assumes only that the intended shifts are finite and that the $m$ th leading principal submatrix of $T$ is nonsingular.

Hence, zero diagonal entries at positions $m+1, \ldots, n-m$ in $T$ do not affect the information contained in the bulge pairs and consequently do not affect the convergence of the QZ iteration. What happens to such a zero diagonal entry if a bulge pair passes through it? This question has been addressed by Ward [46, 47] for $m \leq 2$, and by Watkins for general $m$ [53]. The answer is that the zero diagonal entry moves $m$ positions upward along the diagonal. Note that although it is assumed in [53] that the multishift QZ iteration is based on Givens rotations, the same answer holds for a QZ iteration based on (opposite) Householder matrices; see [31].

These results imply that infinite eigenvalues need only be deflated if they correspond to zero diagonal entries at positions $1, \ldots, m$ and $n-m+1, \ldots, n$ of $T$. Other zero diagonal entries will be automatically moved upward in the course of the QZ algorithm to the top diagonal positions, where they then can be deflated. Note, however, that this "transport" of zero diagonal elements holds only under the assumption of exact arithmetic; it can be severely affected by roundoff error. 
Example 4.2. Consider the $10 \times 10$ matrix pair

$$
(H, T)=\left(\left[\begin{array}{ccccc}
3 & 3 & \cdots & \cdots & 3 \\
1 & 3 & \ddots & \ddots & \vdots \\
& \ddots & \ddots & \ddots & \vdots \\
& & \ddots & 3 & 3 \\
& & & 1 & 3
\end{array}\right],\left[\begin{array}{ccccc}
1 & 1 & \cdots & \cdots & 1 \\
& 0 & \ddots & & \vdots \\
& & \ddots & \ddots & \vdots \\
& & & 0 & 1 \\
& & & & 1
\end{array}\right]\right)
$$

in Hessenberg-triangular form. It can be shown that this matrix pair has four infinite eigenvalues. Applying a single-shift QZ iteration, Algorithm 1 with $m=1$, once to $(H, T)$ leads to an updated triangular matrix $T$ with the leading diagonal entry being exactly zero. None of the other diagonal entries, however, satisfies the deflation criterion (4.2). Also in the course of further QZ iterations applied to the deflated matrix pair no other infinite eigenvalue can be detected. After convergence, the three remaining infinite eigenvalues of $(H, T)$ have been perturbed to finite eigenvalues of magnitude $\approx 1.9 \times 10^{5}$. On the other hand, if all entries of $T$ satisfying (4.2) are subsequently deflated before any QZ iteration, then all four infinite eigenvalues are detected.

Example 4.2 reveals that not taking care of all (nearly) zero diagonal entries in $T$ increases the chances that infinite eigenvalues go undetected. Besides the obvious disadvantages, failing to detect infinite eigenvalues may have an adverse effect on the convergence of the QZ algorithm [29, 53]. There is no simple cure for the effects observed in Example 4.2. Setting a diagonal entry, which is known to be zero in exact arithmetic but does not satisfy (4.2), explicitly to zero would spoil the backward stability of the QZ algorithm. We therefore recommend taking care of all nearly zero diagonal entries in $T$ before applying a QZ iteration. Small or - in rare circumstances - even zero diagonal entries in $T$ may still appear during a multishift QZ iteration. In particular, we may encounter such a situation when having chased some but not all of the bulge pairs from a chain of bulge pairs. Then the small diagonal entry resides between two smaller chains and from the point of view of Example 4.2 it would be desirable to deflate the corresponding (nearly) infinite eigenvalue. However, with the existing deflation techniques, this can only be achieved by chasing off at least one of the smaller chains.

5. Singular and nearly singular pencils. For a moment, let us consider a square singular pencil $\beta A-\alpha B$. Then the generalized Schur form $(S, T)$ of $(A, B)$ must (in theory) have at least one pair $\left(s_{i i}, t_{i i}\right)$ with $s_{i i}=t_{i i}=0$. This situation appears, for example, when $A$ and $B$ have a common column (or row) null space. On the other hand, given a singular pair $(S, T)$ in generalized Schur form with a regular part, an equivalence transformation of $(S, T)$ that produces upper triangular matrices may give no information about the regular part by inspection of the diagonal elements. For example, the pair

$$
(S, T)=\left(\left[\begin{array}{ll|l|l}
3 & 1 & 0 & 0 \\
0 & 3 & 0 & 0 \\
\hline 0 & 0 & 2 & 0 \\
\hline 0 & 0 & 0 & 0
\end{array}\right],\left[\begin{array}{ll|l|l}
1 & 0 & 0 & 0 \\
0 & 1 & 0 & 0 \\
\hline 0 & 0 & 1 & 0 \\
\hline 0 & 0 & 0 & 0
\end{array}\right]\right)
$$

has the finite eigenvalues $3 / 1,3 / 1$, and $2 / 1$, besides the singular part $(0 / 0)$. The equivalent matrix pair

$$
(S Q, T Q)=\left(\left[\begin{array}{c|cc|c}
0 & 3 & 1 & 0 \\
0 & 0 & 3 & 0 \\
\hline 0 & 0 & 0 & 2 \\
\hline 0 & 0 & 0 & 0
\end{array}\right],\left[\begin{array}{l|ll|l}
0 & 1 & 0 & 0 \\
0 & 0 & 1 & 0 \\
\hline 0 & 0 & 0 & 1 \\
\hline 0 & 0 & 0 & 0
\end{array}\right]\right) \text {, with } Q=\left[\begin{array}{llll}
0 & 1 & 0 & 0 \\
0 & 0 & 1 & 0 \\
0 & 0 & 0 & 1 \\
1 & 0 & 0 & 0
\end{array}\right]
$$


has all diagonal elements equal to zero $(0 / 0)$. So examination of the diagonal elements only gives no indication of the well-defined regular part of $(S, T)$.

In practice, the QZ algorithm will in general not detect the above-mentioned singularities reliably and otherwise well-conditioned eigenvalues can change drastically, meaning that the values of the computed pairs $\left(s_{i i}, t_{i i}\right)$ cannot be trusted (e.g., see Wilkinson [54] for several illustrative examples.) Moreover, it is impossible to decide just by inspection whether $s_{i i}=\epsilon_{1}$ and $t_{i i}=\epsilon_{2}$, with $\epsilon_{1}$ and $\epsilon_{2}$ tiny, correspond to a finite eigenvalue $\epsilon_{1} / \epsilon_{2}$ or to a true singular pencil. Anyhow, with this information we know that $\beta A-\alpha B$ is close to a singular pencil. (Note that the converse of this statement is not true $[9,27]$.)

Although the QZ algorithm delivers erratic results for singular or almost singular cases, the computed results are still exact for small perturbations of the original matrix pair $(A, B)$. To robustly deal with such cases, it is recommended to first identify any singularity and deflate the associated Kronecker structure of $(A, B)$ in a preprocessing step before the QZ algorithm is applied. As with infinite eigenvalues, this can be done by exploiting staircase-type algorithms like GUPTRI [13, 14].

6. Aggressive early deflation applied to the QZ algorithm. The idea behind the aggressive early deflation strategy in the QZ algorithm is to enhance the deflation strategy described in section 3.3 by taking advantage of perturbations outside the subdiagonal entries of the Hessenberg matrix, as in the QR algorithm [6]. This gives the possibility to identify and deflate converged eigenvalues much earlier than either of the deflation criteria (3.2) and (3.3) would do, which results in fewer QZ iterations and thereby has the potential to save both floating point operations and execution time.

6.1. Pairs of reducing perturbations. For simplicity, we consider an $n \times n$ complex unreduced Hessenberg-triangular matrix pair $(H, T)$. Let $P_{H}$ and $P_{T}$ be complex perturbation matrices. Suppose there exist a unitary matrix $Q$ of the form $Q=\left[\begin{array}{cc}1 & 0 \\ 0 & \tilde{Q}\end{array}\right]$ and a unitary matrix $Z$ such that the transformed perturbed matrix pair,

$$
(\hat{H}, \hat{T}) \equiv Q^{H}\left(H+P_{H}, T+P_{T}\right) Z,
$$

is in reduced Hessenberg-triangular form:

$$
\hat{H}=\left[\begin{array}{cc}
\hat{H}_{11} & \hat{H}_{12} \\
0 & \hat{H}_{22}
\end{array}\right], \quad \hat{T}=\left[\begin{array}{cc}
\hat{T}_{11} & \hat{T}_{12} \\
0 & \hat{T}_{22}
\end{array}\right] .
$$

Then, in analogy to the matrix case, $\left(P_{H}, P_{T}\right)$ is called a reducing perturbation pair. If the norm of $\left(P_{H}, P_{T}\right)$ is tiny, the equivalence transformation above has split the problem of computing the eigenvalues of $(H, T)$ in two (or more) subproblems of smaller size without affecting the backward stability of the QZ algorithm.

In the following, we derive results that characterize and identify pairs of reducing perturbations, which are extensions of similar results for the matrix case [6].

Lemma 6.1. Let $(H, T)$ with $H, T \in \mathbb{C}^{n \times n}$ be in unreduced Hessenberg-triangular form and $P_{H}, P_{T} \in \mathbb{C}^{n \times n}$. Assume that $T+P_{T}$ is invertible. Then $\left(P_{H}, P_{T}\right)$ is a reducing perturbation pair for $(H, T)$ if and only if the regular matrix pair $(H+$ $\left.P_{H}, T+P_{T}\right)$ has a left (generalized) eigenvector $y \in \mathbb{C}^{n}$ with a zero first component, $y_{1}=0$.

Proof. Assume $\left(P_{H}, P_{T}\right)$ is a reducing perturbation pair for $(H, T)$; i.e., there exist a unitary matrix $Q=\left[\begin{array}{cc}1 & 0 \\ 0 & \tilde{Q}\end{array}\right]$ and a unitary matrix $Z$ such that $(\hat{H}, \hat{T})$ defined 
by $(6.1)$ is in reduced block-triangular form (6.2). This implies that $(\hat{H}, \hat{T})$ has a left (generalized) eigenvector $\hat{y}$ with $\hat{y}_{1}=0$; indeed, the first $\operatorname{dim}\left(\hat{H}_{11}\right)$ components equal to zero. Since $Q$ has block diagonal structure, it follows that $y=Q \hat{y}$ is a left eigenvector of $\left(H+P_{H}, T+P_{T}\right)$ with $y_{1}=\hat{y}_{1}=0$.

In the opposite direction, assume that $\left(H+P_{H}, T+P_{T}\right)$ has a left (generalized) eigenvector $y \in \mathbb{C}^{n}$ with a zero first component, $y_{1}=0$, associated with the eigenvalue pair $(\alpha, \beta) \in \mathbb{C}^{2}$, i.e., $\beta y^{H}\left(H+P_{H}\right)=\alpha y^{H}\left(T+P_{T}\right)$. By replacing the initial QR factorization of $B$ in the standard algorithm for reducing a matrix pair $(A, B)$ to Hessenberg-triangular form [18, Alg. 7.7.1] by an RQ factorization of $B$, we construct unitary matrices $Q=\left[\begin{array}{cc}1 & 0 \\ 0 & \tilde{Q}\end{array}\right]$ and $Z$ such that $Q^{H}\left(H+P_{H}, T+P_{T}\right) Z=(\hat{H}, \hat{T})$ is in Hessenberg-triangular form. It follows that $\hat{y}=Q^{H} y$ is a left (generalized) eigenvector of $(\hat{H}, \hat{T})$ and $\hat{y}_{1}=y_{1}=0$ due to the fact that $Q$ is block diagonal. Let $k$ be the smallest index for which $\hat{y}_{k} \neq 0$ and partition $\hat{y}^{H}=[0, z]^{H}$ with $z \in \mathbb{C}^{n-k+1}$. If $\hat{H}$ and $\hat{T}$ are conformably partitioned,

$$
\hat{H}=\left[\begin{array}{cc}
\hat{H}_{11} & \hat{H}_{12} \\
h_{k, k-1} e_{1} e_{k-1}^{T} & \hat{H}_{22}
\end{array}\right], \quad \hat{T}=\left[\begin{array}{cc}
\hat{T}_{11} & \hat{T}_{12} \\
0 & \hat{T}_{22}
\end{array}\right],
$$

then $\beta \hat{y}^{H} \hat{H}=\alpha \hat{y}^{H} \hat{T}$ yields

$$
\beta\left[\hat{h}_{k, k-1} \hat{y}_{k} e_{k-1}^{T}, z^{H} \hat{H}_{22}\right]=\alpha\left[0, z^{H} \hat{T}_{22}\right] .
$$

The nonsingularity of $T+P_{T}$ implies $\beta \neq 0$, which in turn gives $\hat{h}_{k, k-1}=0$; i.e., $\hat{H}$ is in reduced Hessenberg form.

Note that the second part of the proof of Lemma 6.1 also shows how orthogonal matrices $Q$ and $Z$ yielding a deflated matrix pair (6.2) can be obtained by a slightly modified form of Hessenberg-triangular reduction. In the context of aggressive deflation, a useful reducing perturbation pair $\left(P_{H}, P_{T}\right)$ must have enough zero structure so that relatively little work is needed to retransform $\left(H+P_{H}, T+P_{T}\right)$ to Hessenbergtriangular form. By restricting $P_{H}$ and $P_{T}$ to Hessenberg and triangular matrices, respectively, there will be no extra work.

LEMma 6.2. $\left(P_{H}, P_{T}\right)$ is a reducing perturbation pair for $(H, T)$ of minimal Frobenius norm in the set of Hessenberg-triangular pairs if and only if $P_{T}$ is the zero matrix and $P_{H}$ is zero except for some subdiagonal entry.

Proof. Let $\left(P_{H}, P_{T}\right)$ be a reducing perturbation pair in Hessenberg-triangular form. Decompose $P_{H}=P_{H}^{(s)}+P_{H}^{(u)}$ in its subdiagonal part $P_{H}^{(s)}$ and its upper triangular part $P_{H}^{(u)}$. Then $\left(P_{H}^{(s)}, 0\right)$ is a reducing perturbation pair of smaller norm.

This choice leads to the small-subdiagonal deflation strategy for the QZ algorithm described in section 3.3.

In order to reach a more aggressive deflation strategy, we must allow more general perturbations, where $\left(P_{H}, P_{T}\right)$ is not necessarily in Hessenberg-triangular form. Extending the matrix case, we consider small perturbations $P_{H}$ and $P_{T}$ that are nonzero only in the last $k$ rows and $k+1$ columns. Now, if $k \ll n$, the cost is small (compared to a QZ iteration) to retransform the perturbed matrix pair to Hessenberg-triangular form; see also section 6.2. 
Let the matrix pair $(H, T)$ be in unreduced Hessenberg-triangular form with $H, T \in \mathbb{C}^{n \times n}$ and partitioned as follows:

$$
H=\left[\begin{array}{ccc}
H_{11} & H_{12} & H_{13} \\
H_{21} & H_{22} & H_{23} \\
0 & H_{32} & H_{33}
\end{array}\right], \quad T=\left[\begin{array}{ccc}
T_{11} & T_{12} & T_{13} \\
0 & T_{22} & T_{23} \\
0 & 0 & T_{33}
\end{array}\right],
$$

where the block rows from top to bottom (and block columns from left to right) have $n-n_{w}-1,1$, and $n_{w}$ rows (columns), respectively. Let the perturbation pair $\left(P_{H}, P_{T}\right)$ be partitioned conformably with $(H, T)$, but with the following nonzero structure:

$$
P_{H}=\left[\begin{array}{ccc}
0 & 0 & 0 \\
0 & 0 & 0 \\
0 & P_{32}^{(H)} & P_{33}^{(H)}
\end{array}\right], \quad P_{T}=\left[\begin{array}{ccc}
0 & 0 & 0 \\
0 & 0 & 0 \\
0 & 0 & P_{33}^{(T)}
\end{array}\right] .
$$

A special choice of such perturbations is given in the following lemma.

Lemma 6.3. If $(\alpha, \beta)$ is an eigenvalue pair of $\left(H_{33}, T_{33}\right)$ with left eigenvector $y$, normalized such that $\|y\|_{2}=1$, then $\left(P_{H}, P_{T}\right)$ partitioned as in (6.4) with $P_{32}^{(H)}=$ $-\left(y^{H} H_{32}\right) y, P_{33}^{(H)}=0$, and $P_{T}=0$ is a reducing perturbation pair.

Proof. We have

$$
\beta\left[0,0, y^{H}\right]\left(H+P_{H}\right)=\beta\left[0,0, y^{H} H_{33}\right]=\alpha\left[0,0, y^{H} T_{33}\right]=\alpha\left[0,0, y^{H}\right] T .
$$

This shows that $\left[0,0, y^{H}\right]^{H}$ is a left eigenvector of the perturbed matrix pair $(H+$ $\left.P_{H}, T+P_{T}\right)$ with $P_{T}=0$, which together with Lemma 6.1 concludes the proof.

To search for reducing perturbation pairs, we can choose from all, generically $n_{w}$, possible perturbations in the sense of Lemma 6.3. Although this strategy will generally yield only a reducing perturbation of approximately minimal Frobenius norm among all pairs of the form (6.4), the perturbations of Lemma 6.3 have the major advantage of being effectively computed and tested. Finding the minimum among all reducing perturbations of the form (6.4) is closely related to finding the distance to uncontrollability of a descriptor system [10]. This connection along with numerical methods for computing the distance to uncontrollability will be studied in a forthcoming paper. However, in preliminary numerical experiments with the multishift QR algorithm we observed that rarely can any extra deflations be gained from using perturbations more general than those of Lemma 6.3.

To illustrate the effectiveness of Lemma 6.3, let us consider the following matrix pair, which has been considered in [1] as an extension of the motivating example in [6]:

$$
\left.(H, T)=\left(\begin{array}{cccccc}
6 & 5 & 4 & 3 & 2 & 1 \\
0.001 & 1 & 0 & 0 & 0 & 0 \\
& 0.001 & 2 & 0 & 0 & 0 \\
& & 0.001 & 3 & 0 & 0 \\
& & & 0.001 & 4 & 0 \\
& & & & 0.001 & 5
\end{array}\right],\left[\begin{array}{llllll}
1 & 1 & 1 & 1 & 1 & 1 \\
& 1 & 0 & 0 & 0 & 0 \\
& & 1 & 0 & 0 & 0 \\
& & & 1 & 0 & 0 \\
& & & & 1 & 0 \\
& & & & & 1
\end{array}\right]\right)
$$

Let us consider a partitioning of the form (6.3) for $n_{w}=5$. Then the eigenvalues of $\left(H_{33}, T_{33}\right)$ are given by the $(\alpha, \beta)$ pairs $(1,1),(2,1), \ldots,(5,1)$ with $\lambda=\alpha / \beta$. Each of these eigenvalues yields a reducing perturbation pair in the sense of Lemma 6.3. The respective norms of $\left\|P_{32}^{(H)}\right\|$ are as follows:

$$
1: 1.0 \times 10^{-3}, 2: 1.0 \times 10^{-6}, 3: 5.0 \times 10^{-10}, 4: 1.7 \times 10^{-13}, 5: 4.2 \times 10^{-17} .
$$

In double precision, the eigenvalue 5 can thus be safely deflated. In single precision, even three eigenvalues $(3,4$, and 5$)$ correspond to a reducing perturbation of norm below machine precision. See also section 7.3, where this example is studied for larger matrices. 
6.2. Implementation aspects. In the following, we describe an efficient method which puts the aggressive early deflation motivated by Lemma 6.3 into practice. For this purpose, we consider a partition of $(H, T)$ of the form (6.3) and focus on the $n_{w} \times\left(n_{w}+1\right)$ submatrix pair $\left(\left[H_{32}, H_{33}\right],\left[0, T_{33}\right]\right)$, which defines the deflation window.

First, by means of the QZ algorithm, orthogonal matrices $Q_{1}$ and $Z_{1}$ resulting in a generalized Schur decomposition of $\left(H_{33}, T_{33}\right)$ are computed. This admits the following partitioning:

$$
Q_{1}^{T}\left(\left[H_{32}, H_{33}\right],\left[0, T_{33}\right]\right)\left[\begin{array}{cc}
1 & 0 \\
0 & Z_{1}
\end{array}\right]=\left(\left[\begin{array}{ccc}
s_{3} & \tilde{H}_{33} & \tilde{H}_{34} \\
s_{4} & 0 & \tilde{H}_{44}
\end{array}\right],\left[\begin{array}{ccc}
0 & \tilde{T}_{33} & \tilde{T}_{34} \\
0 & 0 & \tilde{T}_{44}
\end{array}\right]\right)
$$

where $s_{3}, s_{4}$ are column vectors of appropriate size, and $\left(\tilde{H}_{44}, \tilde{T}_{44}\right)$ is either $1 \times 1$, representing a real eigenvalue of $\left(H_{33}, T_{33}\right)$, or $2 \times 2$, representing a complex conjugate pair of eigenvalues. If $\left(\tilde{H}_{44}, \tilde{T}_{44}\right)$ represents a real eigenvalue, then the corresponding reducing perturbation in the sense of Lemma 6.3 is obtained by setting the scalar $s_{4}$ to zero. Similarly, if $\left(\tilde{H}_{44}, \tilde{T}_{44}\right)$ is $2 \times 2$, a reducing perturbation is obtained by setting the two entries of $s_{4}$ to zero. This is, strictly speaking, not a perturbation in the sense of Lemma 6.3 and it may happen that one of the two complex conjugate eigenvalues of $\left(\tilde{H}_{44}, \tilde{T}_{44}\right)$ considered individually yields a reducing perturbation which is significantly smaller than $\left\|s_{4}\right\|_{2}$. However, deflating this eigenvalue alone is not possible without leaving the realm of real matrices.

There are several possible choices for criteria under which $\left\|s_{4}\right\|_{2}$, the norm of the reducing perturbation described above, can be considered negligible. A liberal deflation criterion, which just preserves numerical backward stability, is given by

$$
\left\|s_{4}\right\|_{2} \leq \mathbf{u}\|H\|_{F} .
$$

A more conservative criterion in the spirit of (3.3) is given by

$$
\left\|s_{4}\right\|_{2} \leq \begin{cases}\mathbf{u}\left|\tilde{H}_{44}\right| & \text { if } \tilde{H}_{44} \text { is } 1 \times 1 \\ \mathbf{u} \sqrt{\left|\operatorname{det}\left(\tilde{H}_{44}\right)\right|} & \text { otherwise }\end{cases}
$$

This is preferred for reasons explained in section 3.3. A range of other criteria can be found in $[6$, sec. 2.4$]$.

If $\left\|s_{4}\right\|_{2}$ satisfies the chosen deflation criterion, we mark $\left(\tilde{H}_{44}, \tilde{T}_{44}\right)$ as deflatable and apply the described process again to the reduced matrix pair $\left.\left(\left[s_{3}, \tilde{H}_{33}\right], \tilde{T}_{33}\right]\right)$. Otherwise, we mark $\left(\tilde{H}_{44}, \tilde{T}_{44}\right)$ as undeflatable and reorder the generalized Schur decomposition of $\left(H_{33}, T_{33}\right)$ to construct orthogonal matrices $Q_{2}$ and $Z_{2}$ such that

$$
\left(Q_{1} Q_{2}\right)^{T}\left(\left[H_{32}, H_{33}\right],\left[0, T_{33}\right]\right)\left[\begin{array}{cc}
1 & 0 \\
0 & Z_{1} Z_{2}
\end{array}\right]=\left(\left[\begin{array}{ccc}
\bar{s}_{3} & \bar{H}_{33} & \bar{H}_{34} \\
\bar{s}_{4} & 0 & \bar{H}_{44}
\end{array}\right],\left[\begin{array}{ccc}
0 & \bar{T}_{33} & \bar{T}_{34} \\
0 & 0 & \bar{T}_{44}
\end{array}\right]\right)
$$

where $\left(\bar{H}_{33}, \bar{T}_{33}\right)$ is of the same order and has the same eigenvalues as $\left(\tilde{H}_{44}, \tilde{T}_{44}\right)$. In this case, the described process is applied again to the matrix pair $\left.\left(\left[\bar{s}_{4}, \bar{H}_{44}\right], \bar{T}_{44}\right]\right)$. The whole procedure is repeated until the matrix pair vanishes, i.e., $n_{w}-k$ undeflatable and $k$ deflatable eigenvalues have been found yielding a decomposition of the form

$$
Q^{T}\left(\left[H_{32}, H_{33}\right],\left[0, T_{33}\right]\right)\left[\begin{array}{ll}
1 & 0 \\
0 & Z
\end{array}\right]=\left(\left[\begin{array}{ccc}
\check{s}_{3} & \check{H}_{33} & \check{H}_{34} \\
\check{s}_{4} & 0 & \check{H}_{44}
\end{array}\right],\left[\begin{array}{ccc}
0 & \check{T}_{33} & \check{T}_{34} \\
0 & 0 & \check{T}_{44}
\end{array}\right]\right)
$$


where $\left(\check{H}_{44}, \check{T}_{44}\right)$ is $k \times k$ and contains all deflatable eigenvalues. Moreover, we have $\left\|\check{s}_{4}\right\|_{2} \leq \sqrt{k} \mathbf{u}\|H\|_{F}$ no matter whether (6.6) or (6.7) is used. Hence, $\check{s}_{4}$ can be safely set to zero and the QZ algorithm is continued with the $(n-k) \times(n-k)$ matrix pair

$$
(\tilde{H}, \tilde{T})=\left(\left[\begin{array}{ccc}
H_{11} & H_{12} & H_{13} Z \\
H_{21} & H_{22} & H_{23} Z \\
0 & \check{s}_{3} & \check{H}_{33}
\end{array}\right],\left[\begin{array}{ccc}
T_{11} & T_{12} & T_{13} Z \\
0 & T_{22} & T_{23} Z \\
0 & 0 & \check{T}_{33}
\end{array}\right]\right) .
$$

Note that the matrix pair $(\tilde{H}, \tilde{T})$ is not in Hessenberg-triangular form due to the "spike" $\check{s}_{3}$. If we apply a Householder matrix $\mathcal{H}_{1}\left(\check{s}_{3}\right)=I-\beta v v^{T}$ to the last $n_{w}-k$ rows of $(\tilde{H}, \tilde{T})$, we have

$$
\mathcal{H}_{1}\left(\check{s}_{3}\right) \check{T}_{33}=\check{T}_{33}-\beta v\left(\check{T}_{33}^{T} v\right)^{T} .
$$

Hence, $\mathcal{H}_{1}\left(\check{s}_{3}\right) \check{T}_{33}$ is a rank-one update of a triangular matrix. Similar to updating algorithms for the QR decomposition [18, sec. 12.5], we can construct an orthogonal matrix $Z_{3}$ as a sequence of $n_{w}-k-1$ Givens rotations such that $Z_{3}^{T} \check{T}_{33}^{T} v=\gamma e_{n}$ for some $\gamma \in \mathbb{R}$. Consequently,

$$
\mathcal{H}_{1}\left(\check{s}_{3}\right) \check{T}_{33} Z_{3}=\check{T}_{33} Z_{3}-\beta \gamma v e_{n}^{T}
$$

is an upper Hessenberg matrix. By another sequence of $n_{w}-k-1$ Givens rotations the subdiagonal elements of $\mathcal{H}_{1}\left(\check{s}_{3}\right) \check{T}_{33} Z_{3}$ can be eliminated so that $\mathcal{H}_{1}\left(\check{s}_{3}\right) \check{T}_{33} Z_{3} Z_{4}$ becomes upper triangular. The described algorithm requires $\mathcal{O}\left(\left(n_{w}-k\right)^{2}\right)$ flops which is favorable compared to the $\mathcal{O}\left(\left(n_{w}-k\right)^{3}\right)$ flops needed for computing an RQ factorization of $\mathcal{H}_{1}\left(\check{s}_{3}\right) \check{T}_{33}$ from scratch. Finally, the standard reduction algorithm [18, Alg. 7.7.1] without the initial QR factorization is applied to the matrix pair $\mathcal{H}_{1}\left(\check{s}_{3}\right)\left(\check{H}_{33}, \check{T}_{33}\right) Z_{3} Z_{4}$ in order to compute orthogonal matrices $Q_{3}=\left[\begin{array}{cc}1 & 0 \\ 0 & Q_{3}\end{array}\right]$ and $Z_{5}$ such that $Q_{3}^{T} \mathcal{H}_{1}\left(\check{s}_{3}\right)\left(\check{H}_{33}, \check{T}_{33}\right) Z_{3} Z_{4} Z_{5}$ is Hessenberg-triangular. Setting

$$
\tilde{Q}=\left[\begin{array}{cc}
I_{n-n_{w}} & 0 \\
0 & \mathcal{H}_{1}\left(\check{s}_{3}\right) Q_{3}
\end{array}\right], \quad \tilde{Z}=\left[\begin{array}{cc}
I_{n-n_{w}} & 0 \\
0 & Z_{3} Z_{4} Z_{5}
\end{array}\right]
$$

yields a Hessenberg-triangular matrix pair $\tilde{Q}^{T}(\tilde{H}, \tilde{T}) \tilde{Z}$ from which the multishift QZ algorithm can be continued. Note that before continuing with a multishift QZ iteration it can be beneficial to apply aggressive early deflation again if sufficiently many eigenvalues have been deflated, i.e., if the ratio $k / n_{w}$ is above a certain threshold, which has been set to $40 \%$ in our experiments (parameter \#3 in Table 7.1).

7. Computational experiments. To assess their performance and robustness, we have implemented the newly developed variants of the QZ algorithm in FORTRAN 77 and performed several experiments to be described in the following subsections.

7.1. Computational platform(s). The experiments are carried out on one processor of two of the HPC2N clusters, seth and sarek, which have advanced memory systems with different characteristics.

The cluster seth consists of 120 nodes, dual Athlon MP2000+ (1.667Ghz) with 1 GB memory per node. Athlon MP2000+ has a $64 \mathrm{kB}$ instruction, a $64 \mathrm{kB}$ data L1 Cache, and $256 \mathrm{kB}$ of integrated L2 cache. Software used: Debian GNU/Linux 3.0, Portland F90 6.0, ATLAS BLAS 3.5.9.

The cluster sarek consists of 190 HP DL145 nodes, with dual AMD Opteron 248 $(2.2 \mathrm{GHz})$ and $8 \mathrm{~GB}$ memory per node. AMD Opteron 248 has a $64 \mathrm{kB}$ instruction 
TABLE 7.1

Default values for some parameters of the multishift QZ algorithm with aggressive early deflation.

\begin{tabular}{llcc}
\hline & & seth & sarek \\
\hline$\# 1$ & Minimal (sub)matrix pair dimension for multishift QZ iterations & 300 & 300 \\
$\# 2$ & Minimal (sub)matrix pair dimension for aggressive early deflation & 300 & 300 \\
\#3 & Minimal success rate for repeated aggressive early deflation & $40 \%$ & $40 \%$ \\
$\# 4$ & Window size for simultaneous deflation of infinite eigenvalues & 60 & 84 \\
\#5 & Number of infinite eigenvalues to be deflated simultaneously & 20 & 28 \\
\hline
\end{tabular}

and $64 \mathrm{kB}$ data L1 Cache (2-way associative) and a $1024 \mathrm{kB}$ unified L2 Cache (16-way associative). Software used: Debian GNU/Linux 3.1, Portland F90 6.0, Goto BLAS 0.94 . $\left.10^{-16}\right)$.

All results reported are run in double precision real arithmetic $\left(\epsilon_{\text {mach }} \approx 2.2 \times\right.$

7.2. Random matrix pairs. The described multishift QZ iterations and deflation algorithms depend on various parameters, which all have some influence on the overall execution time of the QZ algorithm. We have performed numerical experiments with randomly generated matrix pairs and numerous sets of parameters to measure the influence of each individual parameter. In the following, we focus on the three parameters that have been observed to have the largest impact on the execution time and therefore require particular attention:

$m$ : number of simultaneous shifts used in each multishift QZ iteration (integer multiple of $n_{s}$ ),

$n_{s}$ : number of shifts contained in each bulge during multishift QZ iterations,

$n_{w}$ : aggressive early deflation window size.

All other parameters turned out to have less influence on the performance and have been set in a heuristic manner. The default values displayed in Table 7.1 yielded good performance for matrix pairs of size $500, \ldots, 3000$. If the order of an active submatrix pair in the course of the multishift QZ algorithm described in section 3 becomes smaller than parameter \#1, it is more efficient to resort to double-shift QZ iterations. Similarly, if the order is smaller than parameter \#2, aggressive early deflation is turned off. It is best to choose \#1 not smaller than \#2. If aggressive early deflation yielded the deflation of $k$ eigenvalues and the ratio $k / n_{w}$ exceeds parameter \#3, another search for early deflations is immediately performed on the deflated matrix pair before applying a (multishift) QZ iteration. Finally, the parameters \#4 and \#5 represent the window size and the maximal number of infinite eigenvalues to be pushed simultaneously in the block algorithm for deflating infinite eigenvalues described in section 4.3. It is necessary to choose \#4 larger than two times \#5; we found choosing \#4 three times larger nearly optimal.

To make the new implementation better comparable to the LAPACK version 3.0 implementation, we used throughout all experiments the liberal deflation criteria (3.2), (4.2), and (6.6). The use of the more conservative deflation criteria (3.3) and (6.7) may result in more accurate eigenvalues but may also lead to slightly more QZ iterations.

7.2.1. Influence of $\boldsymbol{m}$ and $\boldsymbol{n}_{\boldsymbol{s}}$. To measure the influence of the parameters $m$ and $n_{s}$ on the performance of the multishift QZ algorithm without aggressive early deflation, we generated $n \times n$ matrices $A$ and $B$ having pseudorandom entries uniformly distributed in the interval $[-1,1]$ and reduced them to Hessenberg-triangular form by applying the LAPACK version 3.0 routine DGGHRD. Figures 7.1 and 7.2 display 

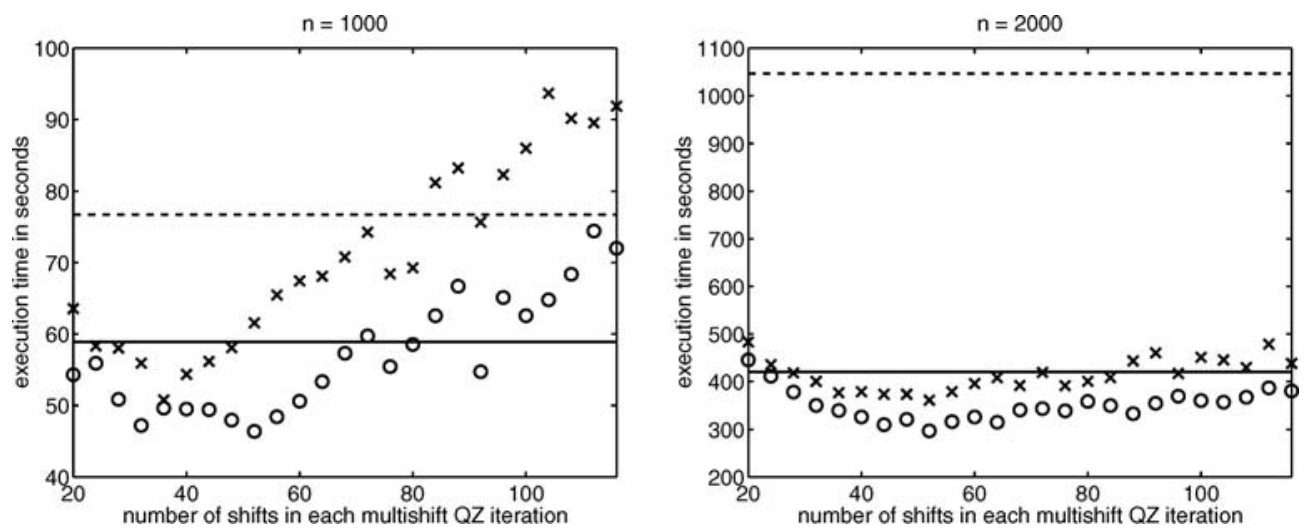

FIG. 7.1. Random matrix pairs: Execution times on seth of DHGEQZ (dashed line), KDHGEQZ (solid line), and MULTIQZ without aggressive early deflation for $n_{s}=2$ (crosses) and $n_{s}=4$ (circles).
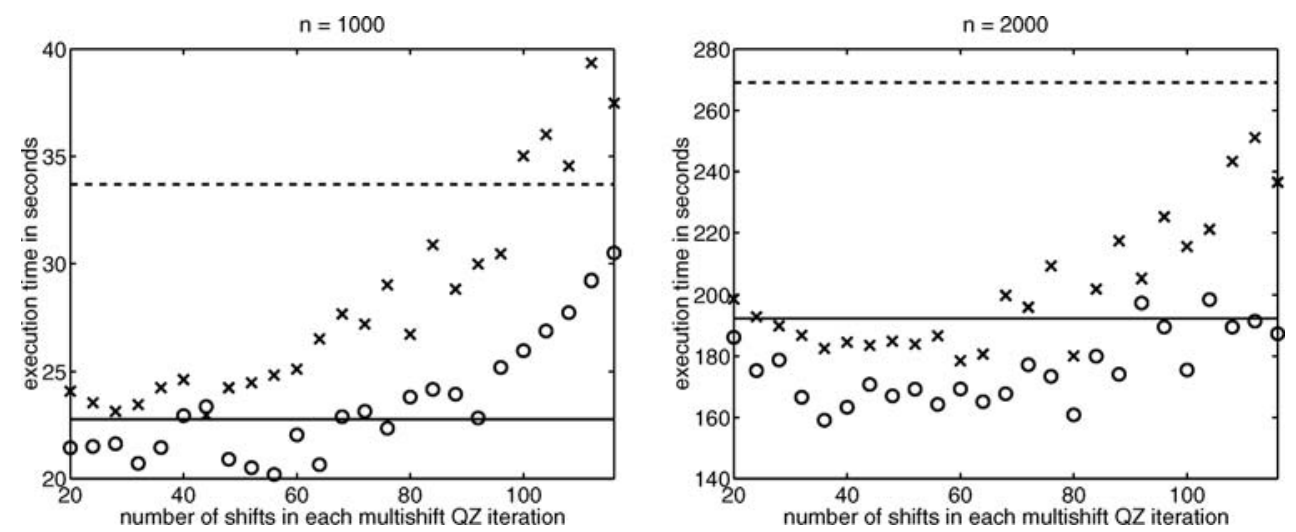

FIG. 7.2. Random matrix pairs: Execution times on sarek of DHGEQZ (dashed line), KDHGEQZ (solid line), and MULTIQZ without aggressive early deflation for $n_{s}=2$ (crosses) and $n_{s}=4$ (circles).

the measured execution times for the following implementations of the QZ algorithm:

DHGEQZ: LAPACK version 3.0 implementation as described in the original paper by Moler and Stewart [37] with some of the modifications proposed in $[29,47,49]$; see also section 2.1 .

KDHGEQZ: Blocked variant of DHGEQZ, developed by Dackland and Kågström [12].

MULTIQZ: Multishift QZ algorithm based on tightly coupled chains of tight bulges as described in section 3 .

The graphs in Figures 7.1 and 7.2 show the sensitivity of the measured execution times for $n=1000$ and $n=2000$ as a function of $m$, the degree of the multishift polynomial used in MULTIQZ, where $m$ is varying between 20 and 116 with step size 4 . Note that in these and the following figures all results for a fixed value of $n$ are observations from a single random matrix pair. On seth it can be observed that the optimal time for MULTIQZ is significantly lower for both $n_{s}=2$ and $n_{s}=4$ than the time needed by DHGEQZ and KDHGEQZ. On sarek the gained savings are less substantial. In fact, for $n_{s}=2$ and $n=1000$ even with the optimal $m$, MULTIQZ requires slightly more execution time than KDHGEQZ. 

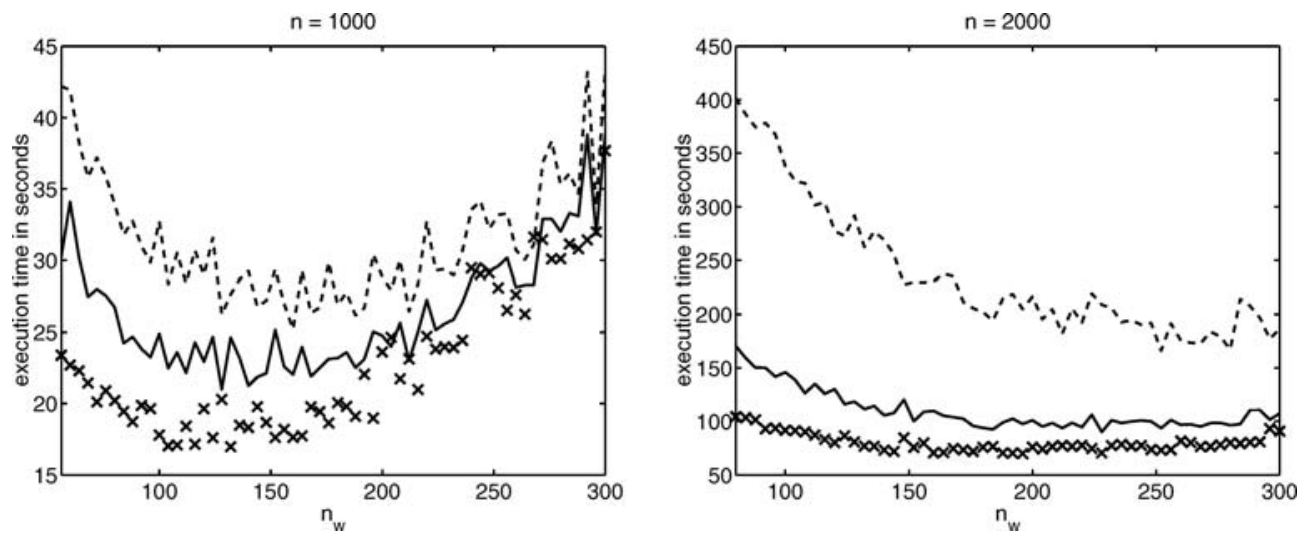

FIG. 7.3. Random matrix pairs: Execution times on seth of DHGEQZ with aggressive early deflation (dashed line), KDHGEQZ with aggressive early deflation (solid line), and MULTIQZ with aggressive early deflation (crosses).
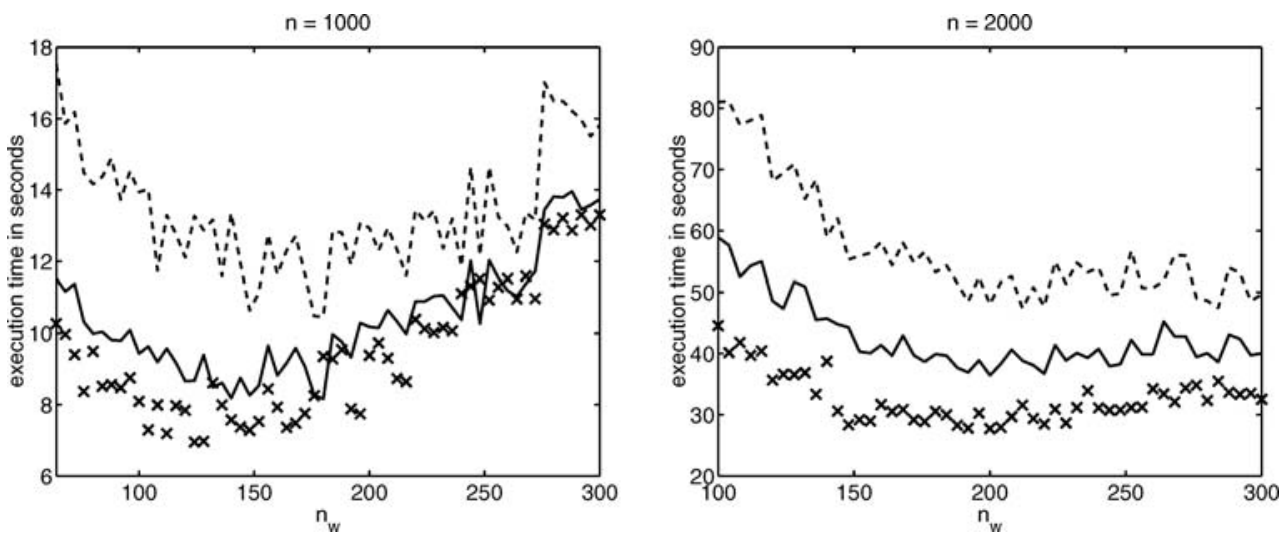

FIG. 7.4. Random matrix pairs: Execution times on sarek of DHGEQZ with aggressive early deflation (dashed line), KDHGEQZ with aggressive early deflation (solid line), and MULTIQZ with aggressive early deflation (crosses).

7.2.2. Influence of $\boldsymbol{n}_{\boldsymbol{w}}$. Similar results for the three implementations of the QZ algorithm with aggressive early deflation are displayed in Figures 7.3 and 7.4. The graphs show the sensitivity of the measured execution times with respect to $n_{w}$, the size of the deflation window, for $n=1000$ and $n=2000$. For DHGEQZ and KDHGEQZ aggressive early deflation has not been performed after each QZ iteration but only after every $m / 2$ (double-shift) QZ iterations, meaning that an overall number of $m$ shifts is applied before each search for early deflations. For all three implementations, we have chosen the optimal value for $m$ in the set $\{20,24, \ldots, 116\}$. Moreover, we have set $n_{s}=4$ for MULTIQZ. Again, it can be seen that MULTIQZ outperforms LAPACK's DHGEQZ, but is also faster than KDHGEQZ.

7.2.3. Infinite eigenvalues. To generate matrix pairs having large numbers of infinite eigenvalues, we generated Hessenberg-triangular matrix pairs $(H, T)$ in the same manner as in the previous two subsections and set each diagonal element of $T$ with probability 0.5 to zero. For $n=2000$, this resulted in a matrix pair $(H, T)$ with roughly 1000 zero entries on the diagonal of $T$. Either implementation of the 
TABLE 7.2

Infinite eigenvalues: Execution times in seconds on seth and sarek of MULTIQZ with aggressive early deflation for a $2000 \times 2000$ random matrix pair with 656 infinite eigenvalues. The numbers shown in brackets correspond to the part (number between 0 and 1) of the execution time that was spent for deflating infinite eigenvalues.

\begin{tabular}{lrc}
\hline \multicolumn{1}{c}{ Deflation strategy } & \multicolumn{1}{c}{ seth } & sarek \\
\hline All $\infty$ eigenvalues at top left corner (unblocked) & $204.8(0.70)$ & $71.6(0.66)$ \\
All $\infty$ eigenvalues at top left corner (blocked) & $142.1(0.56)$ & $59.8(0.54)$ \\
All $\infty$ eigenvalues at nearest corner (unblocked) & $137.0(0.60)$ & $43.5(0.55)$ \\
All $\infty$ eigenvalues at nearest corner (blocked) & $91.6(0.45)$ & $37.1(0.44)$ \\
Necessary $\infty$ eigenvalues at nearest corner (unblocked) & $99.6(0.11)$ & $43.9(0.13)$ \\
Necessary $\infty$ eigenvalues at nearest corner (blocked) & $94.1(0.05)$ & $42.8(0.11)$ \\
\hline
\end{tabular}

QZ algorithm detected 656 infinite eigenvalues. If only those infinite eigenvalues that correspond to nearly zero diagonal entries at the top left and bottom right corners of $T$ are deflated in the course of the QZ algorithm (see section 4.4), then a significant portion remains undetected. For example, when using this strategy together with DHGEQZ only 399 infinite eigenvalues were detected, which confirms the findings of Example 4.2. On the other hand, this strategy significantly lowers the time spent for dealing with infinite eigenvalues. This can be seen in the last two rows of Table 7.2, which lists execution times for various strategies used in MULTIQZ with $n_{s}=4$ and the optimal values for $m$ and $n_{w}$ obtained from section 7.2.2. Note, however, that failing to detect infinite eigenvalues adversely affects the convergence of the QZ algorithm; the time spent for QZ iterations increases from 61 to 89 seconds on seth. We remark that the use of the windowing technique described in section 4.3 is denoted by "(blocked)" in Table 7.2. There are other interesting observations in the figures of this table. Deflating infinite eigenvalues at the nearest corner of the matrix $T$ (and not at only one corner as it is done in DHGEQZ) is a simple means to significantly lower the execution time. Roughly the same portion of time can be saved by using the windowing technique. The most efficient strategy, which detects all 656 infinite eigenvalues, is a combination of both techniques, deflation at the nearest corner combined with windowing.

7.3. Aggressive early deflation at its best. In exceptional cases, aggressive early deflation can have a dramatic positive effect on the computational time. Such a case are matrix pairs of the form (6.5), for which rarely any QZ iterations are needed to deflate eigenvalues. The graphs in Figure 7.5 show the measured execution times of the three implementations of the QZ algorithm with and without aggressive early deflation for $n=600$ to 3000 (seth) or 4000 (sarek) with step size 200. For all examples we used $n_{w}=n-1$.

We remark that since aggressive early deflation works so well, the time spent for (multishift) QZ iterations is negligible compared to the overall time. In fact, the timings for DHGEQZ, KDHGEQZ, and MULTIQZ with aggressive early deflation are virtually identical and orders of magnitude better than without early deflation. For example, for $n=4000$ the time of DHGEQZ is reduced from nearly one hour to less than 7 seconds.

7.4. Examples from applications. The purpose of this section is to summarize the performance of the multishift QZ algorithm with aggressive early deflation for matrix pairs that arise from practically relevant applications. We have selected 16 matrix pairs from the Matrix Market collection [3], 6 matrix pairs from model reduction benchmark collections $[11,30]$, and 4 matrix pairs arising from the com- 

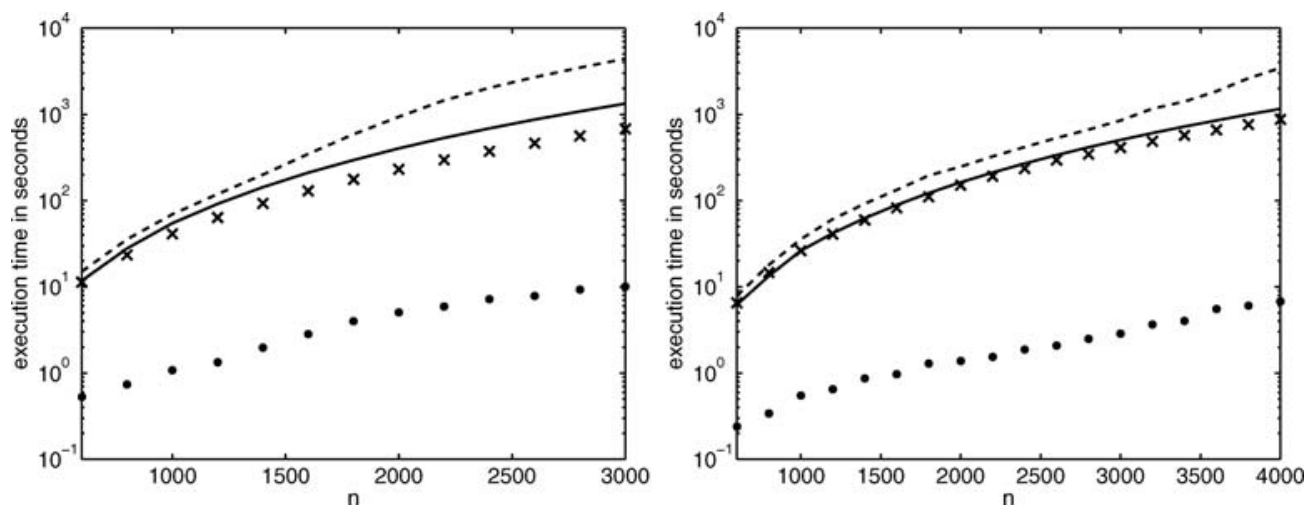

FIG. 7.5. BBM/ADK example $[1,6]$ : Execution times (in logarithmic scale) on seth (left figure) and sarek (right figure) of DHGEQZ (dashed line), KDHGEQZ (solid line), MULTIQZ (crosses) without aggressive early deflation, and DHGEQZ/KDHGEQZ/MULTIQZ with aggressive early deflation (dots).

putation of corner singularities of elliptic PDEs [38]. A more detailed description of the selected matrix pairs along with individual performance results can be found in Appendix A of the technical report version [22] of this paper. In the following, we summarize these results and sort the matrix pairs into groups according to their order $n$ as shown in the following table.

\begin{tabular}{l|cccc}
\hline Group & G1 & G2 & G3 & G4 \\
order & $n \in[485,1000]$ & $n \in[1001,1500]$ & $n \in[1501,2000]$ & $n \in[2001,3600]$ \\
\#pairs & 6 & 8 & 5 & 7 \\
\hline
\end{tabular}

Matrix pairs arising from applications differ in many aspects from random matrix pairs. An aspect which can particularly affect the performance of the QZ algorithm is that the matrix entries in most of the pairs from the Matrix Market collection differ wildly in magnitude. Bad scaling makes the performance of the QZ algorithm erratic and much less predictive than for random matrix pairs. For example, LAPACK's DHGEQZ requires 338 seconds for a $1900 \times 1900$ matrix pair arising from the discretized heat equation (see section A.19 in [22]) but less than 25 seconds for the $1922 \times 1922$ matrix pair consisting of the matrices BCSSTK26 and BCSSTM26 from the Matrix Market collection (see section A.12 in [22]). Balancing can remedy bad scaling but we have decided not to make use of it since this preprocessing step is by default turned off in most major software environments such as MATLAB.

We have tested DHGEQZ, KDHGEQZ, and MULTIQZ for all possible combinations of the parameters $n_{w}$ (aggressive early deflation window size), $m$ (number of shifts before each aggressive early deflation) and $n_{s}$ (number of shifts per bulge) satisfying $n_{w} \in$ $\{40,60,80, \ldots, 400\}, m \in\{24,32,40, \ldots, 160\}$, and $n_{s} \in\{2,4\}$. Due to the memory limitations of seth, all numerical experiments described in the following have only been performed on sarek. Also, to limit the variety of parameters, we have turned off the blocked algorithms for deflating infinite eigenvalues. Column 3 of Table 7.3 shows for each of the four groups the average times in seconds of DHGEQZ and KDHGEQZ without aggressive early deflation. The fourth column displays the average computing times for all three implementations with aggressive early deflation obtained by choosing $m$ and $n_{w}$ optimally for each matrix pair in the group. The fifth column displays similar times obtained by choosing $m$ and $n_{w}$ optimally to yield the best average performance for all matrix pairs together in each group. The corresponding choices 
TABLE 7.3

Application examples: Summary of measured execution times and choice of parameters $m$ and $n_{w}$ that give optimal average performance.

\begin{tabular}{clrrrrr}
\hline Group & Implementation & W/o agg. & Optimal & Ave. opt. & $m$ & $n_{w}$ \\
\hline G1 & DHGEQZ+AGG & 8.86 & 4.61 & 5.03 & 24 & 120 \\
& KDHGEQZ+AGG & 7.07 & 3.62 & 3.78 & 24 & 100 \\
& MULTIQZ $\left(n_{s}=2\right)+$ AGG & - & 3.36 & 3.45 & 24 & 60 \\
& MULTIQZ $\left(n_{s}=4\right)+$ AGG & - & 3.40 & 3.56 & 24 & 40 \\
\hline G2 & DHGEQZ+AGG & 55.5 & 23.7 & 26.5 & 56 & 180 \\
& KDHGEQZ+AGG & 41.4 & 17.2 & 18.9 & 40 & 140 \\
& MULTIQZ $\left(n_{s}=2\right)+$ AGG & - & 15.3 & 16.5 & 72 & 160 \\
& MULTIQZ $\left(n_{s}=4\right)+$ AGG & - & 15.1 & 16.3 & 88 & 180 \\
\hline G3 & DHGEQZ+AGG & 130.3 & 53.6 & 56.4 & 48 & 220 \\
& KDHGEQZ+AGG & 89.9 & 36.5 & 38.8 & 72 & 200 \\
& MULTIQZ $\left(n_{s}=2\right)+$ AGG & - & 30.3 & 30.7 & 56 & 200 \\
& MULTIQZ $\left(n_{s}=4\right)+$ AGG & - & 27.5 & 30.0 & 88 & 200 \\
\hline G4 & DHGEQZ+AGG & 479 & 157 & 170 & 48 & 340 \\
& KDHGEQZ+AGG & 271 & 97 & 104 & 40 & 220 \\
& MULTIQZ $\left(n_{s}=2\right)+$ AGG & - & 80 & 85 & 72 & 220 \\
& MULTIQZ $\left(n_{s}=4\right)+$ AGG & - & 115 & 124 & 80 & 220 \\
\hline
\end{tabular}

of $m$ and $n_{w}$ are listed in columns 6 and 7, respectively. The difference between the figures of columns 4 and 5 is roughly $10 \%$, which demonstrates that nearly optimal average performance can be obtained without having to optimize $m$ and $n_{w}$ for each matrix pair individually. Ideally, $m$ and $n_{w}$ should be chosen adaptively within the QZ algorithm, but it is not clear how such a strategy can be effectively realized.

On average, the multishift QZ algorithm with aggressive early deflation (MULTIQZ +AGG, $n_{s}=2$ ) is between 2.6 and 6 times faster than the original LAPACK implementation (DHGEQZ). Surprisingly, the block version of Dackland and Kågström is, when equipped with aggressive early deflation (KDHGEQZ+AGG), only $10 \%$ to $20 \%$ slower than the multishift QZ algorithm. There is little justification for setting $n_{s}$, the number of shifts per bulge, to $n_{s}=4$ in favor of $n_{s}=2$, in contrast to the results for random matrix pairs.

We have also tested the backward stability of the new variants of the QZ algorithm by measuring the residual $\left\|\left(\hat{Q}^{T} A \hat{Z}-\hat{S}, \hat{Q}^{T} B \hat{Z}-\hat{T}\right)\right\|_{F}$ of the computed Schur decomposition $(\hat{S}, \hat{T})$ as well as the orthogonality $\left\|\hat{Q}^{T} \hat{Q}-I\right\|_{F},\left\|\hat{Z}^{T} \hat{Z}-I\right\|_{F}$ of the computed transformation matrices $\hat{Q}$ and $\hat{Z}$. The obtained results are of the same order as those obtained using the LAPACK implementation.

8. Conclusions. In this paper, we have developed new multishift variants of the QZ algorithm using advanced deflation techniques which significantly improve upon the performance compared to all existing implementations. It is planned that an implementation of our multishift QZ algorithm with aggressive early deflation is included in a coming release of LAPACK. The ideas presented here are currently applied to the development of a distributed memory QZ algorithm. Future work also includes the investigation of extending the described results to even more general versions of the QR algorithm, such as the periodic QR and QZ algorithms.

9. Final remarks and acknowledgments. The work presented in this article is based on preliminary results derived in $[1,31]$. The authors are greatly indebted to Ralph Byers and David Watkins for several discussions on the odds and ends of multishift QR and QZ algorithms. The computational experiments in section 7 were 
performed using facilities of the High Performance Computing Center North (HPC2N) in Umeå.

\section{REFERENCES}

[1] B. Adlerborn, K. Dackland, And B. KÅgström, Parallel and blocked algorithms for reduction of a regular matrix pair to Hessenberg-triangular and generalized Schur forms, in PARA 2002, J. Fagerholm et al., eds., Lecture Notes in Comput. Sci. 2367, Springer-Verlag, Berlin, 2002, pp. 319-328.

[2] E. Anderson, Z. Bai, C. H. Bischof, S. Blackford, J. W. Demmel, J. J. Dongarra, J. Du Croz, A. Greenbaum, S. Hammarling, A. McKenney, and D. C. Sorensen, LAPACK Users' Guide, 3rd ed., Software Environ. Tools 9, SIAM, Philadelphia, 1999.

[3] Z. Bai, D. Day, J. W. Demmel, and J. J. Dongarra, A Test Matrix Collection for NonHermitian Eigenvalue Problems (Release 1.0), Technical report CS-97-355, Department of Computer Science, University of Tennessee, Knoxville, TN, 1997. Also available online from http://math.nist.gov/MatrixMarket.

[4] Z. Bai and J. W. Demmel, On a block implementation of the Hessenberg multishift $Q R$ iterations, Internat. J. High Speed Comput., 1 (1989), pp. 97-112.

[5] C. H. Bischof, B. LAng, And X. Sun, A framework for symmetric band reduction, ACM Trans. Math. Software, 26 (2000), pp. 581-601.

[6] K. Braman, R. Byers, and R. Mathias, The multishift QR algorithm. Part II: Aggressive early deflation, SIAM J. Matrix Anal. Appl., 23 (2002), pp. 948-973.

[7] K. Braman, R. Byers, and R. Mathias, The multishift $Q R$ algorithm. Part I: Maintaining well-focused shifts and level 3 performance, SIAM J. Matrix Anal. Appl., 23 (2002), pp. 929-947.

[8] K. E. Brenan, S. L. Campbell, and L. R. Petzold, Numerical Solution of Initial-Value Problems in Differential-Algebraic Equations, Classics Appl. Math. 14, SIAM, Philadelphia, 1995.

[9] R. Byers, C. He, And V. Mehrmann, Where is the nearest non-regular pencil?, Linear Algebra Appl., 285 (1998), pp. 81-105.

[10] R. Byers, The descriptor controllability radius, in Proceedings of the Conference on the Mathematical Theory of Networks and Systems, U. Helmke, R. Mennicken, and J. Saurer, eds., MTNS '93, Akademie Verlag, Berlin, 1994, pp. 85-88.

[11] Y. Chahlaoui and P. Van Dooren, A Collection of Benchmark Examples for Model Reduction of Linear Time Invariant Dynamical Systems, SLICOT working note 2002-2, WGS, 2002.

[12] K. DACKLAND AND B. KÅGSTRÖM, Blocked algorithms and software for reduction of a regular matrix pair to generalized Schur form, ACM Trans. Math. Software, 25 (1999), pp. 425454.

[13] J. W. Demmel And B. KÅgström, The generalized Schur decomposition of an arbitrary pencil $A-\lambda B$ : Robust software with error bounds and applications. I. Theory and algorithms, ACM Trans. Math. Software, 19 (1993), pp. 160-174.

[14] J. W. Demmel And B. KÅgström, The generalized Schur decomposition of an arbitrary pencil $A-\lambda B$ : Robust software with error bounds and applications. II. Software and applications, ACM Trans. Math. Software, 19 (1993), pp. 175-201.

[15] J. J. Dongarra, J. Du Croz, I. S. Duff, and S. Hammarling, A set of level 3 basic linear algebra subprograms, ACM Trans. Math. Software, 16 (1990), pp. 1-17.

[16] A. A. Dubrulle, The Multishift QR Algorithm-Is it Worth the Trouble?, Technical report TR 6320-3558, IBM Scientific Center, Palo Alto, CA, 1991.

[17] F.R. Gantmacher, The Theory of Matrices, Chelsea, New York, 1960.

[18] G. H. Golub and C. F. Van Loan, Matrix Computations, 3rd ed., Johns Hopkins University Press, Baltimore, MD, 1996.

[19] K. Goto And R. VAn DE Geijn, High-Performance Implementation of the Level-3 BLAS, Technical report TR-2006-23, Department of Computer Sciences, The University of Texas at Austin, Austin, TX, 2006.

[20] E. Griepentrog And R. MÄrz, Differential-Algebraic Equations and Their Numerical Treatment, Teubner Texte zur Mathematik, Teubner-Verlag, Leipzig, 1986.

[21] N. J. Higham, Accuracy and Stability of Numerical Algorithms, SIAM, Philadelphia, 1996.

[22] B. K̊̊gström And D. Kressner, Multishift Variants of the QZ Algorithm with Aggressive Early Deflation, Report UMINF-05.11, Department of Computing Science, Umeå University, Umeå, Sweden, 2005. Also appeared as LAPACK Working Note 173. 
[23] B. KÅgström, P. Ling, and C. F. Van Loan, GEMM-based level 3 BLAS: Algorithms for the model implementations, ACM Trans. Math. Software, 24 (1999), pp. 268-302.

[24] B. KÅgström, P. Ling, ANd C. F. VAn LOAN, GEMM-based level 3 BLAS: High-performance model implementations and performance evaluation benchmark, ACM Trans. Math. Software, 24 (1999), pp. 303-316.

[25] B. KÅgström AND P. PoromaA, Computing eigenspaces with specified eigenvalues of a regular matrix pair $(A, B)$ and condition estimation: Theory, algorithms and software, Numer. Algorithms, 12 (1996), pp. 369-407.

[26] B. KА̊gTRÖM, A direct method for reordering eigenvalues in the generalized real Schur form of a regular matrix pair $(A, B)$, in Linear Algebra for Large Scale and Real-Time Applications (Leuven, 1992), M. S. Moonen, G. H. Golub, and B. L. R. De Moor, eds., NATO Adv. Sci. Inst. Ser. E Appl. Sci. 232, Kluwer Academic Publishers, Dordrecht, The Netherlands, 1993, pp. 195-218.

[27] B. KÅgström, Singular matrix pencils, in Templates for the Solution of Algebraic Eigenvalue Problems, Z. Bai, J. W. Demmel, J. J. Dongarra, A. Ruhe, and H. van der Vorst, eds., Software Environ. Tools 11, SIAM, Philadelphia, 2000, pp. 260-277.

[28] L. KaUfman, The LZ-algorithm to solve the generalized eigenvalue problem, SIAM J. Numer. Anal., 11 (1974), pp. 997-1024.

[29] L. KAUfman, Some thoughts on the $Q Z$ algorithm for solving the generalized eigenvalue problem, ACM Trans. Math. Software, 3 (1977), pp. 65-75.

[30] J. G. Korvink And B. R. EvGeniI, Oberwolfach benchmark collection, in Dimension Reduction of Large-Scale Systems, P. Benner, V. Mehrmann, and D. C. Sorensen, eds., Lecture Notes Comput. Sci. Eng. 45, Springer-Verlag, Heidelberg, 2005, pp. 311-316.

[31] D. Kressner, Numerical Methods and Software for General and Structured Eigenvalue Problems, Ph.D. thesis, Institut für Mathematik, TU Berlin, Berlin, Germany, 2004.

[32] D. KRESSNER, Block algorithms for reordering standard and generalized Schur forms, LAPACK working note 171, September 2005. ACM Trans. Math. Software, to appear.

[33] D. KREssner, On the use of larger bulges in the QR algorithm, Electron. Trans. Numer. Anal., 20 (2005), pp. 50-63.

[34] V. N. KUblanovSkaya, AB-algorithm and its modifications for the spectral problems of linear pencils of matrices, Numer. Math., 43 (1984), pp. 329-342.

[35] P. Kunkel and V. Mehrmann, Differential-Algebraic Equations. Analysis and Numerical Solution, EMS Publishing House, Zürich, Switzerland, 2006.

[36] B. LANG, Effiziente Orthogonaltransformationen bei der Eigen- und Singulärwertzerlegung, Habilitationsschrift, 1997.

[37] C. B. Moler And G. W. Stewart, An algorithm for generalized matrix eigenvalue problems, SIAM J. Numer. Anal., 10 (1973), pp. 241-256.

[38] C. Pester, CoCoS-Computation of Corner Singularities, preprint SFB393/05-03, Technische Universität Chemnitz, Chemnitz, Germany, 2005.

[39] P. J. Rabier and W. C. Rheinboldt, Nonholonomic Motion of Rigid Mechanical Systems from a DAE Viewpoint, SIAM, Philadelphia, 2000.

[40] G. W. Stewart and J.-G. Sun, Matrix Perturbation Theory, Academic Press, New York, 1990.

[41] G. W. Stewart, On the eigensystems of graded matrices, Numer. Math., 90 (2001), pp. 349370 .

[42] T. StYkel, Balanced truncation model reduction for semidiscretized Stokes equation, Technical report 04-2003, Institut für Mathematik, TU Berlin, Berlin, Germany, 2003.

[43] C. Tischendorf, Solution of Index-2-DAEs and Its Application in Circuit Simulation, Dissertation, Humboldt-Univ. zu Berlin, Berlin, Germany, 1996.

[44] P. VAn Dooren, Algorithm 590: DSUBSP and EXCHQZ: Fortran subroutines for computing deflating subspaces with specified spectrum, ACM Trans. Math. Software, 8 (1982), pp. 376382.

[45] C. F. VAn LoAn, Generalized Singular Values with Algorithms and Applications, Ph.D. thesis, The University of Michigan, Ann Arbor, MI, 1973.

[46] R. C. WARD, A Numerical Solution to the Generalized Eigenvalue Problem., Ph.D. thesis, University of Virginia, Charlottesville, VA., 1974.

[47] R. C. WARD, The combination shift QZ algorithm, SIAM J. Numer. Anal., 12 (1975), pp. 835853.

[48] R. C. WARD, Balancing the generalized eigenvalue problem, SIAM J. Sci. Statist. Comput., 2 (1981), pp. 141-152.

[49] D. S. WATKINS AND L. Elsner, Theory of decomposition and bulge-chasing algorithms for the generalized eigenvalue problem, SIAM J. Matrix Anal. Appl., 15 (1994), pp. 943-967. 
[50] D. S. Watkins, Shifting strategies for the parallel $Q R$ algorithm, SIAM J. Sci. Comput., 15 (1994), pp. 953-958.

[51] D. S. WATKINS, Forward stability and transmission of shifts in the $Q R$ algorithm, SIAM J. Matrix Anal. Appl., 16 (1995), pp. 469-487.

[52] D. S. WATKIns, The transmission of shifts and shift blurring in the $Q R$ algorithm, Linear Algebra Appl., 241/243 (1996), pp. 877-896.

[53] D. S. Watkins, Performance of the $Q Z$ algorithm in the presence of infinite eigenvalues, SIAM J. Matrix Anal. Appl., 22 (2000), pp. 364-375.

[54] J. H. WILKInson, Kronecker's canonical form and the $Q Z$ algorithm, Linear Algebra Appl., 28 (1979), pp. 285-303. 